\title{
Black hole mass estimates in quasars
}

\section{A comparative analysis of high- and low-ionization lines}

\author{
P. Marziani ${ }^{1}$, A. del Olmo ${ }^{2}$, M. A. Martínez-Carballo ${ }^{3}$, M. L. Martínez-Aldama ${ }^{4}$, G. M. Stirpe ${ }^{5}$, C. A. Negrete ${ }^{6, \star}$, \\ D. Dultzin ${ }^{6}$, M. D’Onofrio ${ }^{7, \star \star}$, E. Bon ${ }^{8}$, and N. Bon ${ }^{8}$
}

1 INAF, Osservatorio Astronomico di Padova, vicolo dell' Osservatorio 5, 35122 Padova, Italy e-mail: paola.marziani@oapd.inaf.it

2 Instituto de Astrofisíca de Andalucía, IAA-CSIC, Glorieta de la Astronomia s/n, 18008 Granada, Spain e-mail: chony@iaa.es

3 Departamento de Matemática Aplicada and IUMA, Universidad de Zaragoza, 50009 Zaragoza, Spain e-mail: gelimc@unizar.es

${ }^{4}$ Center for Theoretical Physics, Polish Academy of Science, 02-668 Warsaw, Poland

5 INAF, Osservatorio di Astrofisica e Scienza dello Spazio, 40129 Bologna, Italy

${ }^{6}$ Instituto de Astronomía, UNAM, Mexico D.F. 04510, Mexico

7 Dipartimento di Fisica \& Astronomia "Galileo Galilei", Università di Padova, Vicolo ell’Osservatorio 3, 35122 Padova, Italy

8 Astronomical Observatory, Belgrade, Serbia

Received 11 February 2019 / Accepted 1 May 2019

\begin{abstract}
Context. The inter-line comparison between high- and low-ionization emission lines has yielded a wealth of information on the structure and dynamics of the quasar broad line region (BLR), including perhaps the earliest unambiguous evidence in favor of a disk + wind structure in radio-quiet quasars.

Aims. We carried out an analysis of the CIv $\lambda 1549$ and $\mathrm{H} \beta$ line profiles of 28 Hamburg-ESO high-luminosity quasars and of 48 low- $z$, low-luminosity sources in order to test whether the width of the high-ionization line Civ $\lambda 1549$ could be correlated with $\mathrm{H} \beta$ and be used as a virial broadening estimator.

Methods. We analyze intermediate- to high-S/N, moderate-resolution optical and near-infrared (NIR) spectra covering the redshifted Civ $\lambda 1549$ and $\mathrm{H} \beta$ over a broad range of luminosity $\log L \sim 44-48.5\left[\mathrm{erg} \mathrm{s}^{-1}\right]$ and redshift $(0-3)$, following an approach based on the quasar main sequence.

Results. The present analysis indicates that the line width of Civ $\lambda 1549$ is not immediately offering a virial broadening estimator equivalent to $\mathrm{H} \beta$. At the same time a virialized part of the BLR appears to be preserved even at the highest luminosities. We suggest a correction to FWHM(Civ $\lambda 1549)$ for Eddington ratio (using the Civ $\lambda 1549$ blueshift as a proxy) and luminosity effects that can be applied over more than four dex in luminosity.

Conclusions. Great care should be used in estimating high- $L$ black hole masses $M_{\mathrm{BH}}$ from Civ $\lambda 1549$ line width. However, once a corrected FWHM CIV $\lambda 1549$ is used, a CIV $\lambda 1549$-based scaling law can yield unbiased $M_{\mathrm{BH}}$ values with respect to the ones based on $\mathrm{H} \beta$ with sample standard deviation $\approx 0.3$ dex.
\end{abstract}

Key words. quasars: general - quasars: emission lines - quasars: supermassive black holes - line: profiles - ISM: jets and outflows

\section{Introduction}

Type-1 active galactic nuclei (AGNs) and quasars show the same broad optical-UV lines almost always accompanied by broad permitted FeII emission (e.g., Vanden Berk et al. 2001). However, even among type-1 sources we face a large diversity in observational manifestations involving line profiles, internal line shifts as well as emission line intensity ratios (e.g., Sulentic et al. 2000a; Bachev et al. 2004; Yip et al. 2004; Kuraszkiewicz et al. 2009; Zamfir et al. 2010; Shen \& Ho 2014, and Sulentic \& Marziani 2015 for a recent review). Broad line measurements involving $\mathrm{H} \beta$ line width and FeII strength are not randomly distributed but instead define a quasar "main sequence" (MS; e.g., Boroson \& Green 1992; Sulentic et al. 2000a; Shen \& Ho 2014). The MS can be traced in an optical plane defined by FeII emission prominence and the hydrogen

\footnotetext{
* CONACyT research fellow, Instituto de Astronomía, UNAM.
}

$\star \star$ INAF associate, Osservatorio Astronomico di Padova.
$\mathrm{H} \beta$ line width. The FeII strength is parametrized by the intensity ratio involving the FeII blue blend at $4570 \AA$ and broad $\mathrm{H} \beta$, that is, $R_{\mathrm{FeII}}=\mathrm{I}(\mathrm{FeII} \lambda 4570) / \mathrm{I}(\mathrm{H} \beta)$, and the hydrogen $\mathrm{H} \beta$ line width by its full width at half maximum. Along the MS, sources with higher $R_{\text {FeII }}$ show narrower broad $\mathrm{H} \beta$ (Population A, FWHM $(\mathrm{H} \beta) \lesssim 4000 \mathrm{~km} \mathrm{~s}^{-1}$, Sulentic et al. 2000a). Lower $R_{\mathrm{FeII}}$ is associated with sources with broader $\mathrm{H} \beta$ profiles (Pop. B with $F W H M(\mathrm{H} \beta) \gtrsim 4000 \mathrm{~km} \mathrm{~s}^{-1}$, Sulentic et al. 2011). A glossary of the MS-related terminology is provided in Appendix A.

Studies of the Balmer lines have played a prominent role in characterizing the MS and the properties of the broad-lineemitting region (BLR) in low- $z(\lessgtr 0.8)$ quasars with $\mathrm{H} \beta$ providing information for the largest number of sources (e.g., Osterbrock \& Shuder 1982; Wills et al. 1985; Sulentic 1989; Zamfir et al. 2010; Hu et al. 2012; Steinhardt \& Silverman 2013; Shen 2016, for a variety of observational and statistical approaches). The most important application of the FWHM $\mathrm{H} \beta$ has been its use as a virial broadening estimator (VBE) to derive black hole masses $\left(M_{\mathrm{BH}}\right)$ 
from single-epoch observations of large samples of quasars (e.g., McLure \& Jarvis 2002; McLure \& Dunlop 2004; Vestergaard \& Peterson 2006; Assef et al. 2011; Shen 2013; Peterson 2014, and references therein). The underlying assumption is that the $\mathrm{H} \beta$ line width provides the most reliable VBE, which is likely to be the case, albeit with some caveats (e.g., Trakhtenbrot \& Netzer 2012, see also Shen 2013, Peterson 2014 for reviews).

Balmer lines provide a reliable VBE up to $z \lesssim 2$ (Matsuoka et al. 2013; Karouzos et al. 2015) at cosmic epochs of less than a few gigayears. The importance of having a reliable VBE at even earlier cosmic epochs cannot be underemphasized. The entire scenario of early structure formation is affected by inferences from estimates of quasar black hole masses. Overestimates of $M_{\mathrm{BH}}$ by lines whose broadening is in excess of the virial one can have implications for the quasar mass function. Also, at high redshift $(z \gtrsim 6)$ when the Universe was less than 1 billion years old, overestimates have further implications for the formation and mass spectrum of the seed black holes (Latif \& Ferrara 2016) that may have been responsible, along with Pop. III stars, for the reionization of the process at $z \sim 7-10$ (e.g., Gallerani et al. 2017 , for a review).

Strong and relatively unblended Civ $\lambda 1549$ has been the best candidate for a VBE beyond $z \sim 1.5$, where $\mathrm{H} \beta$ is shifted into the IR domain. CIV $\lambda 1549$ can be observed up to redshift $z \approx 6$ with optical spectrometers, and in the near-infrared (NIR) bands up to redshift $z \approx 7.5$ (Bañados et al. 2018) and beyond. Can Crv $\lambda 1549$ be used as an immediate surrogate for $\mathrm{H} \beta$ when $\mathrm{H} \beta$ is invisible or hard to obtain? Before attempting an answer to this question, two considerations are in order.

First, measurements of the CIv $\lambda 1549$ line profiles remain of uncertain interpretation without a precise determination of the quasar rest frame: an accurate $z$ measurement is not easy to obtain from broad lines, and redshift determinations at $z \gtrsim 1$ from optical survey data suffer systematic biases as large as several hundreds of kilometres per second (Hewett \& Wild 2010; Shen et al. 2016). Reliable studies tie CIV measurements to a rest frame derived from the $\mathrm{H} \beta$ narrow component $(+[\mathrm{O}$ III $] \lambda \lambda 4959,5007$ whenever applicable; e.g., Mejía-Restrepo et al. 2016; for problems in the use of [OIII] $\lambda \lambda 4959,5007$, see Zamanov et al. 2002; Hu et al. 2008).

Second, significant Civ $\lambda 1549$ blueshifts are observed over a broad range in redshift and luminosity, from the nearest Seyfert 1 galaxies to the most powerful radio-quiet quasars (Wills et al. 1993; Sulentic et al. 2007, 2017; Richards et al. 2011; Coatman et al. 2016; Shen 2016; Bischetti et al. 2017; Bisogni et al. 2017; Vietri 2017). Measurements of the Civ $\lambda 1549$ profile velocity displacement provide an additional dimension to a 4D "eigenvector 1" (4DE1) space built on parameters that are observationally independent ("orthogonal") and related to different physical aspects (Sulentic \& Marziani 2015). Inclusion of the Crv $\lambda 1549$ shift as a 4DE1 parameter was motivated by the earlier discovery of internal redshift differences between low- and high-ionization lines (Burbidge \& Burbidge 1967; Gaskell 1982; Tytler \& Fan 1992; Brotherton et al. 1994a; Corbin \& Boroson 1996; Marziani et al. 1996).

The current interpretation of the BLR in quasars sees the broad lines arising in a region that is physically and dynamically composite (e.g., Collin-Souffrin et al. 1988; Elvis 2000; Ferland et al. 2009; Kollatschny \& Zetzl 2013; Grier et al. 2013; Du et al. 2016). Civ $\lambda 1549$ is a doublet originating from an ionic species of ionization potential (IP) four times larger than Hydrogen $(54 \mathrm{eV}$ vs. $13.6 \mathrm{eV}$ ), and is therefore a prototypical high-ionization line (HIL). The line is mainly produced by collisional excitation from the ground state ${ }^{2} \mathrm{~S}_{0}$ to ${ }^{2} \mathrm{~S}_{\frac{1}{2}, \frac{3}{2}}$ at the temperature of photo-ionized
BLR gas $\left(T \sim 10^{4} \mathrm{~K}\right.$, Netzer 1990) in the fully ionized zone of the line-emitting gas. Empirically, the line is relatively strong (rest frame equivalent width $W \sim 10-100 \AA$ depending on the source location on the MS) and only moderately contaminated on the red side (red shelf) by HeII $\lambda 1640$ and OIII] $\lambda 1663$ plus weak emission from FEIUV multiplets (Fine et al. 2010). The Balmer line $\mathrm{H} \beta$ assumed to be representative of the lowionization lines (LILs, from ionic species with IP $\lesssim 20 \mathrm{eV}$ ) is instead enhanced in a partially ionized zone due to the strong $\mathrm{X}$-ray emission of quasars and to the large column density of the line-emitting gas $\left(N_{\mathrm{c}} \gtrsim 10^{23} \mathrm{~cm}^{-2}\right.$; Kwan \& Krolik 1981). Comparison of $\mathrm{H} \beta$ and CIV $\lambda 1549$ profiles in the same sources tells us that they provide independent inputs to BLR models - their profiles can be dissimilar and several properties uncorrelated (see, e.g., Fig. C2 of Mejía-Restrepo et al. 2016).

It is possible to interpret $\mathrm{H} \beta$ and Civ $\lambda 1549$ profiles as associated with two sub-regions within the BLR (e.g., Baldwin et al. 1996; Hall et al. 2003; Leighly 2004; Snedden \& Gaskell 2004; Czerny \& Hryniewicz 2011; Plotkin et al. 2015): one emitting predominantly LILs (e.g., Dultzin-Hacyan et al. 1999; Matsuoka et al. 2008), and a second emitting predominantly HILs, associated with gas outflows and winds (e.g., Richards et al. 2011; Yong et al. 2018). This view is in accordance with early models of the BLR structure involving a disk and outflow or wind component (Collin-Souffrin et al. 1988; Elvis 2000). Intercomparison of $\mathrm{CIV} \lambda 1549$ and $\mathrm{H} \beta$ at low $z$ and moderate luminosity provided the most direct observational evidence that this is the case at least for radio-quiet (RQ) quasars (Corbin \& Boroson 1996; Sulentic et al. 2007; Wang et al. 2011; Coatman et al. 2016). Modeling involves a disk + wind system (e.g., Proga et al. 2000; Proga \& Kallman 2004; Flohic et al. 2012; Sdowski et al. 2014; Vollmer et al. 2018, for different perspectives), although the connection between disk structure and BLR (and hence the $\mathrm{H} \beta$ and CIV $\lambda 1549$ emitting regions) is still unclear.

There are additional caveats, as the CIv $\lambda 1549$ blueshifts are not universally detected. Their amplitude is a strong function of the location along the MS (Sulentic et al. 2000b, 2007; Sun et al. 2018). Large blueshifts are clearly detected in Population A, with sources accreting at relatively high rates, and reach extreme values for quasars at the high- $R_{\text {FeII }}$ end along the MS. In Pop. $\mathrm{B}$, the wind component is not dominating the line broadening of Civ $\lambda 1549$ at moderate luminosity; conversely, the Civ $\lambda 1549$ and $\mathrm{H} \beta$ line profile intercomparison indicates that the dynamical relevance of the Civ $\lambda 1549$ blueshift is small, that is, the ratio between the centroid at half-maximum $c\left(\frac{1}{2}\right)$ and the FWHM is $\ll 1$ (Sulentic et al. 2007). Reverberation mapping studies indicate that the velocity field is predominantly Keplerian (Pei et al. 2017 and references therein for the prototypical source NGC 5548, Denney et al. 2010; Grier et al. 2013), and that the Civ $\lambda 1549$ emitting region is closer to a continuum source than that of $\mathrm{H} \beta$ (e.g., Peterson \& Wandel 2000; Kaspi et al. 2007; Trevese et al. 2014). The issue is complicated by luminosity effects on the Civ $\lambda 1549$ shifts that may have gone undetected at low redshifts. Both Pop. A and B sources at $\log L \gtrsim 47 \mathrm{erg} \mathrm{s}^{-1}$ show large amplitude blueshifts in Civ $\lambda 1549$ (Sulentic et al. 2017; Bisogni et al. 2017; Vietri et al. 2018). The present work considers the trends associated with the MS as well as the luminosity effects that may appear second-order in low-luminosity samples to provide corrections to the FWHM of $\mathrm{H} \beta$ and ultimately a scaling law based on Civ $\lambda 1549$ FWHM and UV continuum luminosity that may be unbiased with respect to $\mathrm{H} \beta$ and with a reasonable scatter. 
The occurrence of Civ $\lambda 1549$ large shifts challenges the suitability of the CIV $\lambda 1549$ profile broadening as a VBE for $M_{\mathrm{BH}}$ estimates (see e.g., Shen 2013, for a review). Results at low redshift suggest that the Civ $\lambda 1549$ line is completely unsuitable for part of Pop. A sources (Sulentic et al. 2007). A similar conclusion was reached at $z \approx 2$ on a sample of 15 high-luminosity quasars (Netzer et al. 2007). More recent work tends to confirm that the CIV $\lambda 1549$ line width is not straightforwardly related to virial broadening (e.g., Mejía-Restrepo et al. 2016). However, the Civ $\lambda 1549$ line is strong and observable up to $z \approx 6$ with optical spectrometers. It is so highly desirable to have a consistent VBE up to the highest redshifts that various attempts (e.g., Brotherton et al. 2015) have been made to rescale Civ $\lambda 1549$ line width estimators to the width of LILs such as $\mathrm{H} \beta$ and $\mathrm{Mg}$ II $\lambda 2800$. Several conflicting claims have recently been made on the valid use of Civ $\lambda 1549$ width in high-redshift quasars (e.g., Assef et al. 2011; Shen \& Liu 2012; Denney et al. 2012; Karouzos et al. 2015; Coatman et al. 2017; Mejía-Restrepo et al. 2018a).

From the previous outline we infer that a proper approach to testing the suitability of the CIv $\lambda 1549$ line width as a VBE is to compare Civ $\lambda 1549$ and $\mathrm{H} \beta$ profiles along the quasar MS, and to extend the luminosity range including intermediate-tohigh redshift ( $\gtrsim 1.4$ ) sources when $\mathrm{H} \beta$ is usually not covered by optical observations. A goal of this paper is to analyze the factors yielding large discrepancies between the $M_{\mathrm{BH}}$ estimates from $\mathrm{H} \beta$ and Civ $\lambda 1549$, with a focus on the aspect and physical factors affecting the broadening of the two lines.

The quasar sample used in the present paper joins two samples with both $\mathrm{H} \beta$ and $\mathrm{CIV} \lambda 1549$ data, one at low luminosity and redshift ( $\lesssim 0.7$, Sulentic et al. 2007), and one at high luminosity, in the range $1.5 \lesssim z \lesssim 3$ presented and analyzed by Sulentic et al. (2017, hereafter Paper I). The sample provides a wide coverage in luminosity and Eddington ratio (Sect. 2); $\mathrm{H} \beta$ line coverage for each Civ $\lambda 1549$ observation; and consistent analysis of the line profiles of both Civ $\lambda 1549$ and $\mathrm{H} \beta$ (Sect. 3). Our approach is intended to overcome some of the sample-dependent difficulties encountered by past studies. Results involve the reduction of the measured Civ $\lambda 1549$ line width to a VBE (Sect. 4) with a correction factor dependent on both shift amplitude and luminosity. They are discussed in terms of BLR structure (Sect. 5.2), and specifically of the interplay between broadening associated with the outflow (very relevant for Civ 21549 ) and with orientation effects (which are dominating for $\mathrm{H} \beta$ ). Finally, a new $M_{\mathrm{BH}}$ scaling law with line width and luminosity (Sect. 5.4) is presented. The new Civ $\lambda 1549$ scaling law, which considers different corrections for Pop. A and B, separately, may provide an unbiased estimator of black hole masses derived from $\mathrm{H} \beta$ over a wide range in luminosity (Sect. 5.5).

\section{Sample}

\subsection{High-luminosity VLT data for Hamburg-ESO quasars}

The high- $L$ quasars considered in the present study are 28 sources identified in the HE survey (Wisotzki et al. 2000, hereafter the HE sample) in the redshift range $1.4 \lesssim z \lesssim 3.1$. All satisfy the conditions on the absolute B magnitude $M_{\mathrm{B}} \lesssim-27.5$ and on the bolometric luminosity $\log L \gtrsim 10^{47.5} \mathrm{erg} \mathrm{s}^{-1}$. They are therefore among the most luminous quasars ever discovered in the Universe, and a relatively rare population even at $z \approx 1-2$ when luminous quasars were more frequent than at low redshift (the luminosity function at $M_{\mathrm{B}} \approx-27.5$ is $\Phi\left(M_{\mathrm{B}}\right) \sim 10^{-8} \mathrm{Mpc}^{-3} \mathrm{mag}^{-1} \mathrm{com}-$ pared to $\sim 10^{-6} \mathrm{Mpc}^{-3} \mathrm{mag}^{-1}$ at $M_{\mathrm{B}} \approx-25$, corresponding to the "knee" of the Boyle et al. 2000 luminosity function).
The Civ $\lambda 1549$ data were obtained with the FORS1 spectrograph at VLT and Dolores at TNG; the matching $\mathrm{H} \beta$ observations with the ISAAC spectrometer were analyzed in detail by Sulentic et al. (2006a). The resolutions at FWHM of the CIV $\lambda 1549$ data are $\lesssim 300 \mathrm{~km} \mathrm{~s}^{-1}$ and $\lesssim 600 \mathrm{~km} \mathrm{~s}^{-1}$ for FORS1 and Dolores, respectively; the $\mathrm{H} \beta$ resolution is $\approx 300 \mathrm{~km} \mathrm{~s}^{-1}$ (Sulentic et al. 2004). Typical signal-to-noise ratio $(\mathrm{S} / \mathrm{N})$ values are $\gtrsim 50$.

Resolution and $\mathrm{S} / \mathrm{N}$ are adequate for a multicomponent nonlinear fitting analysis using the IRAF routine specfit (Kriss 1994), involving an accurate deconvolution of $\mathrm{H} \beta$, [OIII] $\lambda \lambda 4959,5007$, FeII, HeII $\lambda 4686$ in the optical, and of CIV $\lambda 1549$ and HeII $\lambda 1640$ in the UV. The CIv $\lambda 1549$ and $\mathrm{H} \beta$ data and the immediate results of the specfit analysis were reported in Paper I.

\subsection{Low-luminosity CIvג1549 and $\mathrm{H} \beta$ data}

We considered a Faint Object Spectrograph (FOS) sample from Sulentic et al. (2007) as a complementary sample at low- $L$ and low- $z$. For the sake of the present paper, we restrict the FOS sample to 29 Pop. A and 19 Pop. B RQ (48 in total) sources covering the CIv $\lambda 1549$ blend spectral range and with previous measurements for the $\mathrm{H} \beta$ profile and $R_{\mathrm{FeII}}$ (Marziani et al. 2003). The list of sources can be obtained by the cross-correlation of the Sulentic et al. (2007) RQ sources (Kellermann's ratio $\left.\log R_{\mathrm{K}}<1.8\right)$ and the Marziani et al. (2003) catalog on Vizier. We excluded NGC 4395 and NGC 4253 whose luminosities are $\log L \approx 40.4$ and 41.7 [ $\mathrm{erg} \mathrm{s}^{-1}$ ], respectively, thus outlying with respect to the $L$ distribution of the FOS sample. The FOS highresolution grisms yielded an inverse resolution $\lambda / \delta \lambda \sim 1000$, equivalent to typical resolution of the data of Marziani et al. (2003). The $\mathrm{S} / \mathrm{N}$ is above $\gtrsim 20$ for both the optical and UV lowredshift data. The FOS sample has a typical bolometric luminosity $\log L \sim 45.2\left[\mathrm{erg} \mathrm{s}^{-1}\right]$ and a redshift $z \lesssim 0.5$.

\subsection{Joint $H E+F O S$ sample}

The HE+FOS sample therefore has 76 sources, of which 43 are Pop. A and 33 Pop. B. The distribution of $\log L$ for the 76 sources of the joint sample (derived from the rest-frame luminosity at $1450 \AA$, assuming a constant bolometric correction equal to 3.5) uniformly covers the range 44-48.5, with similar distributions for Pop. A and B (lower panel of Fig. 1; a K-S test confirms that the two distributions are not significantly different). The Eddington ratio $\left(L / L_{\text {Edd }}\right)$ covers the range $0.01-1$ which means complete coverage of the $L / L_{\text {Edd }}$ range where most sources in optically selected samples are found.

\section{Emission line profile analysis for the FOS+HE sample}

\subsection{Line modeling and measured parameters}

In the following we consider the merit of $\mathrm{H} \beta$ and Civ $\lambda 1549$ as VBEs. Previous work has shown that the $\mathrm{H} \beta$ and $\mathrm{MgII} \lambda 2800$ profiles are almost equally reliable estimators of the "virial" broadening in samples of moderate-to-high luminosity (e.g., Wang et al. 2009; Trakhtenbrot \& Netzer 2012; Shen \& Liu 2012; Marziani et al. 2013a, excluding the MgII $\lambda 2800$ extreme Population A that is significantly broadened by a blueshifted component; Marziani et al. 2013a). However, the broad $\mathrm{H} \beta$ line full profile is often affected by asymmetries toward the line base and by significant line centroid shifts. Typically, the $\mathrm{H} \beta$ line profiles are characterized by two main asymmetries, differentially 


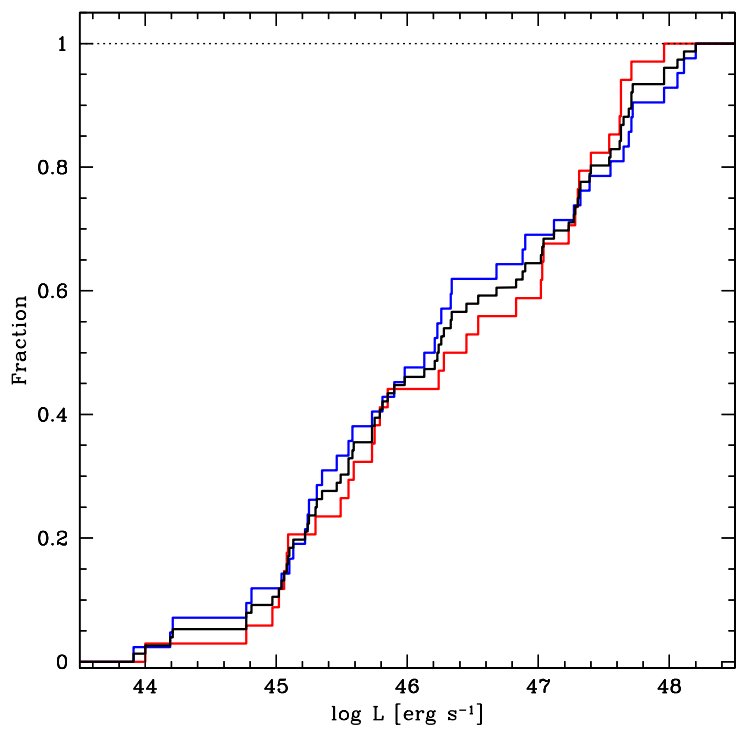

Fig. 1. Cumulative distribution of bolometric luminosity $L$ of the FOS+HE sample (black), Pop. A (blue), and Pop. B (red).

affecting sources in spectral types along the MS (Table A.1 provides the definition of spectral types):

- Pop. A: a blueshifted excess, often modeled with a blueward asymmetric Gaussian component (BLUE) related to the outflows strongly affecting the Civ $\lambda 1549$ and [OIII] $\lambda \lambda 4959,5007$ line profiles (e.g., Negrete et al. 2018, Paper I, and references therein).

- Pop. B: a redward asymmetry modeled with a broader redshifted $\left(F W H M \sim 10000 \mathrm{~km} \mathrm{~s}^{-1}, c\left(\frac{1}{2}\right) \sim 2000 \mathrm{~km} \mathrm{~s}^{-1}\right)$ Gaussian. The very broad Gaussian is meant to represent the innermost part of the BLR, providing a simple representation of the radial stratification of the BLR in Pop. B suggested by reverberation mapping (e.g., Snedden \& Gaskell 2007). This component (hereafter the very broad component, VBC) has been associated with a physical region of high-ionization virialized and closest to the continuum source (Peterson \& Ferland 1986; Brotherton et al. 1994b; Sulentic et al. 2000c; Snedden \& Gaskell 2007; Wang \& Li 2011). While the properties of the very broad line region (VBLR) remain debatable, a decomposition of the full $\mathrm{H} \beta$ profile into a symmetric, unshifted $\mathrm{H} \beta$ component $\left(\mathrm{H} \beta_{\mathrm{BC}}\right)$ and a $\mathrm{H} \beta_{\mathrm{VBC}}$ provides an excellent fit to most $\mathrm{H} \beta$ Pop. B profiles (Sulentic et al. 2002; Zamfir et al. 2010).

Figures 4 and 5 of Paper I show the $\mathrm{H} \beta$ and Civ $\lambda 1549$ profiles of the HE sample, and their multicomponent interpretation. To extract a symmetric, unshifted component that excludes the blueshifted excess and the VBC, we considered a model of the broad $\mathrm{H} \beta$ and $\mathrm{CIV} \lambda 1549$ line with the following components (see also Table A.2):

- Pop. A H $\beta$ and Civ $\lambda 1549$ : an unshifted Lorentzian profile $\left(\mathrm{H} \beta_{\mathrm{BC}}\right)+$ one or more asymmetric Gaussians to model the blueward excess (BLUE).

- Pop. B H $\beta$ and Civ $\lambda 1549$ : an unshifted Gaussian $\left(\mathrm{H} \beta_{\mathrm{BC}}\right)+$ a redshifted $\mathrm{VBC}$ for $\mathrm{H} \beta\left(\mathrm{H} \beta_{\mathrm{VBC}}\right)$. In the $\mathrm{H} \beta$ case, there is no evidence of a blueward excess even at the highest luminosity. However, among Pop. B sources of the HE sample, a prominent CIV $\lambda 1549$ BLUE appears, implying an intensity ratio $\operatorname{Crv} \lambda 1549 / \mathrm{H} \beta \gg 1$ in the BLUE component. The Civ $\lambda 1549$ BLUE is usually fainter in the low-luminosity FOS sample Paper I.

In the fits, narrow components of both $\mathrm{H} \beta\left(\mathrm{H} \beta_{\mathrm{NC}}\right)$ and Civ $\lambda 1549$ (Civ $\lambda 1549_{\text {NC }}$ ) were included. In the case of Civ $\lambda 1549$, separation of the broad and narrow component is subject to significant uncertainty, meaning that the effect of the Civ $\lambda 1549_{\mathrm{NC}}$ needs to be carefully considered (see discussion in Sect. 3.2).

The decomposition approach summarized above has a heuristic value, as the various components are not defined on the basis of a physical model, even if the assumptions on line shapes follow from MS trends. The distinction between BC and VBC might be physically motivated (the emitting region associated with the $\mathrm{BC}$ is the one predominantly emitting FeII), but the decomposition into two symmetric Gaussians is a crude approximation at best. Full profile measurements are added to avoid any exclusive dependence of the results on the profile decomposition. The full profiles of $\mathrm{H} \beta$ and CIV $\lambda 1549$ are parameterized by the FWHM, an asymmetry index (AI), and centroid at $\frac{1}{2}$ and $\frac{1}{4}$ peak fractional intensity, $c\left(\frac{1}{2}\right)$ and $c\left(\frac{1}{4}\right)$. The definition of centroids and AI follows Zamfir et al. (2010):

$$
\begin{aligned}
& c\left(\frac{i}{4}\right)=\frac{\lambda_{\mathrm{B}}\left(\frac{i}{4}\right)+\lambda_{\mathrm{R}}\left(\frac{i}{4}\right)}{2 \lambda_{0}} c, i=1,2,3 ; \frac{i}{4}=0.9, \\
& \text { A.I. }=\frac{\lambda_{\mathrm{B}}\left(\frac{1}{4}\right)+\lambda_{\mathrm{R}}\left(\frac{1}{4}\right)-2 \lambda_{\mathrm{P}}}{\lambda_{\mathrm{B}}\left(\frac{1}{4}\right)+\lambda_{\mathrm{B}}\left(\frac{1}{4}\right)},
\end{aligned}
$$

where $\lambda_{\mathrm{P}}$ is the peak wavelength, and $\lambda_{\mathrm{B}}$ and $\lambda_{\mathrm{R}}$ are the wavelengths on the blue and red side of the line at the $i / 4$ fractional intensities. The centroids are referred to the quasar rest frame, while the AI is referred to as the peak of the line that may be shifted with respect to rest frame. A proxy to $\lambda_{\mathrm{P}}$ which is used in this paper is $\tilde{\lambda}_{\mathrm{P}} \approx \lambda_{0}(1+c(0.9) / c)$.

We assume that the symmetric and unshifted $\mathrm{H} \beta_{\mathrm{BC}}$ and CIv $\lambda 1549_{\mathrm{BC}}$ are the representative line components of the virialized part of the BLR. It is expedient to define a parameter $\xi$ as follows:

$\xi_{\text {line }}=\frac{F W H M_{\text {vir }}}{F W H M}$,

where the $F W H M_{\text {vir }}$ is the FWHM of the "virialized" component, in the following assumed to be $\mathrm{H} \beta_{\mathrm{BC}}$, and the FWHM is the FWHM measured on the full profile (i.e., without correction for asymmetry and shifts). The $\xi$ parameter is a correction factor that can be defined also using components of different lines, for instance CIV $\lambda 1549$ full profile FWHM and $\mathrm{H} \beta_{\mathrm{BC}}$, where $\mathrm{H} \beta_{\mathrm{BC}}$ is assumed to be a reference VBE.

\subsection{The CIVג1549 narrow component in the HE sample and its role in FWHM CIVג1549 ${ }_{B C}$ estimates}

In only two cases does Civ $\lambda 1549_{\mathrm{NC}}$ contribute to the total Civ $\lambda 1549$ flux of the HE Pop. B sources by more than $10 \%$ : HE2202-2557 and HE2355-4621 (Pop. B, Fig. 5 of Paper I). There is no evidence for a strong NC in the HE Pop. A sources except for HE0109-3518 where I(CIv $\left.\lambda 1549_{\mathrm{NC}}\right) \lesssim 0.09$ of the total line flux and whose $\operatorname{Crv} \lambda 1549$ profile resembles those of low-redshift sources that are less luminous by 2-3 dex (the HE0109-3518 Civ $\lambda 1549$ profile is shown in Fig. 4 of Paper I).

In general, considering $\mathrm{H} \beta_{\mathrm{BC}}$ as a reference for Pop. B sources, and comparing FWHM $\mathrm{H} \beta_{\mathrm{BC}}$ to FWHM Civ $\lambda 1549$ with and without removing the Crv $\lambda 1549_{\mathrm{NC}}$ (i.e., to FWHM Civ $\lambda 1549_{\mathrm{BC}}$ and FWHM Crv $\left.\lambda 1549_{\mathrm{BC}}+\operatorname{Crv} \lambda 1549_{\mathrm{NC}}\right)$, the Civ $\lambda 1549_{\mathrm{NC}}$ removal improves the agreement with FWHM $\mathrm{H} \beta_{\mathrm{BC}}$ in five out of six cases when $\operatorname{CIV} \lambda 1549_{\mathrm{NC}}$ has an appreciable effect on the line width (in the other eight cases there is no effect because Civ $\lambda 1549_{\mathrm{NC}}$ is too weak). The FWHMs measured 
on the Civ $\lambda 1549$ profiles without removing the Civ $\lambda 1549_{\mathrm{NC}}$ (i.e. FWHM CIv $\left.\lambda 1549_{\mathrm{BC}}+\operatorname{Civ} \lambda 1549_{\mathrm{NC}}\right)$ are on average $\approx-4 \%$ and $-11 \%$ of the FWHM Civ $\lambda 1549$, for Pop. A and B, respectively. Therefore, (1) subtracting the CIV $\lambda 1549_{\mathrm{NC}}$ improves the agreement between $\mathrm{H} \beta$ and CIv $\lambda 1549$ FWHM; and (2) the average effect is too small to affect our inferences concerning the CIv $\lambda 1549$ line width as a VBE in the HE sample. The CIV $\lambda 1549_{\mathrm{NC}}$ has always been included as an independent component in the line profile fitting of Paper I, following an approach consistently applied to the low-redshift FOS sample and described by Sulentic et al. (2007).

\section{Results}

\section{1. $H \beta$ in the HE sample}

We considered several different measurements of the $\mathrm{H} \beta$ width following empirical corrections derived from previous work on low-redshift samples:

- Substitution of the $\mathrm{H} \beta_{\mathrm{BC}}$ extracted through the specfit analysis in place of the full $\mathrm{H} \beta$ profile.

In principle, extraction of the $\mathrm{H} \beta_{\mathrm{BC}}$ should be the preferred approach, and the FWHM $\mathrm{H} \beta_{\mathrm{BC}}$ the preferred VBE. To test the reliability of the FWHM values, we performed Monte-Carlo repetitions of the $\mathrm{H} \beta$ fit for Pop. B sources with the broadest lines $\left(F W H M \mathrm{H} \beta_{\mathrm{BC}} \sim 7000 \mathrm{~km} \mathrm{~s}^{-1}\right.$, and $F W H M \mathrm{H} \beta_{\mathrm{VBC}} \sim$ $11000 \mathrm{~km} \mathrm{~s}^{-1}$ ), assuming an $S / N \approx 20^{1}$, weak and relatively broad [OIII] $\lambda \lambda 4959,5007$, a changing noise pattern, and initial values of the fitting. The values of FWHM $\mathrm{H} \beta_{\mathrm{BC}}$ and $\mathrm{H} \beta_{\mathrm{VBC}}$ were chosen to represent the broadest lines, where FWHM $\mathrm{H} \beta_{\mathrm{BC}}$ measurements might be affected by a degeneracy in the $\mathrm{BC}+\mathrm{VBC}$ decomposition. The dispersion of the Monte Carlo FWHM distribution is almost symmetric, and implies typical FWHM $\mathrm{H} \beta_{\mathrm{BC}}$ uncertainties $\approx 10 \%$ at $1 \sigma$ confidence level. Therefore, the blending should not be a source of strong bias or of large uncertainties in the $\mathrm{H} \beta_{\mathrm{BC}}$ and $\mathrm{H} \beta_{\mathrm{VBC}} \mathrm{FWHM}^{2}$. However, we still expect that in the case of very broad profiles, and low $\mathrm{S} / \mathrm{N}$ or low dispersion, the decomposition of the $\mathrm{H} \beta$ profile into $\mathrm{H} \beta_{\mathrm{BC}}$ and $\mathrm{H} \beta_{\mathrm{VBC}}$ is subject to large uncertainties that are difficult to quantify. To retrieve information on the $\mathrm{H} \beta_{\mathrm{BC}}$ we introduce several corrections that can be applied to the full $\mathrm{H} \beta$ profile without any multicomponent fitting (which also renders the results modeldependent).

- Symmetrization of the full profile: $F W H M_{\text {symm }}=$ $F W H M--2 c\left(\frac{1}{2}\right)$ (symm in Fig. 2). The physical explanation behind the symmetrization approach involves an excess radial velocity on the red side that may be due to gas with a radial infall velocity component, with velocity increasing toward the central black hole (e.g., Wang et al. 2017, and references therein). Generally speaking, redward displacements of line profiles have been explained by invoking a radial infall component plus obscuration (Hu et al. 2008; Ferland et al. 2009);

- Substitution of the FWHM $\mathrm{H} \beta_{\mathrm{BC}}$ with the FWHM measured on the full broad profile of $\mathrm{H} \beta$, corrected according to its spectral type. The spectral types have been assigned following Sulentic et al. (2002, for a conceptually equivalent approach see Shen \& Ho 2014). The corrections are as defined from the

$\mathrm{S} / \mathrm{N}$ is measured per pixel on the continuum.

2 If $S / N$ is relatively high $(\gtrsim 20)$ only in some peculiar cases might the uncertainty be significantly larger. For example, if the $\mathrm{H} \beta$ profile is composed for a narrower core and a broader base, the FWHM measure is unstable, and may abruptly change depending on continuum placement. analysis of the $\mathrm{H} \beta$ profile in a large SDSS-based sample at $0.4 \lesssim z \lesssim 0.7$ (labeled as st in Fig. 2). In practice, this means to correct $\mathrm{H} \beta$ for Pop. B sources by a factor $\xi_{\mathrm{H} \beta} \approx 0.8$ (Marziani et al. $2013 \mathrm{~b})$ and extreme population $\mathrm{A}$ sources $\left(R_{\mathrm{FeII}} \geq 1\right)$ by a factor $\xi_{\mathrm{H} \beta} \approx 0.9$. On average, spectral types A1 and A2 show symmetric profiles for which $\xi_{\mathrm{H} \beta} \approx 1$. Recent work confirmed that the effect of a blueshifted excess on the full profile of $\mathrm{H} \beta$ is small at half-maximum, $0.9 \lesssim \xi_{\mathrm{H} \beta} \lesssim 1.0$ (Negrete et al. 2018). We assume $\xi_{\mathrm{H} \beta}=0.9$ as an average correction for ST A3 and A4. The ratio we derive between $\mathrm{BC}$ and full profile FWHM of HE Pop. $\mathrm{B} \mathrm{H} \beta$ is $\approx 0.82 \pm 0.09$, consistent with the same ratio estimates at moderate luminosity (Marziani et al. 2013b). The st correction can be summarized as follows:

\begin{tabular}{lc}
\hline $\mathrm{ST}$ & $\xi_{\mathrm{H} \beta}$ \\
\hline$A 3-A 4$ & 0.9 \\
$A 1-A 2$ & 1.0 \\
$B 1-B 1+$ & 0.8 \\
\hline
\end{tabular}

- Correction of the width of the full broad $\mathrm{H} \beta$ profile based on the one derived at low $z$ by pairing the observed full broad $\mathrm{H} \beta$ FWHM to the best width estimator from reverberation mapping, following the relation $F W H M_{\mathrm{c}} \approx 1.14$ FWHM-601 $0.0000217 \mathrm{FWHM}^{2}$ derived by Sulentic et al. (2006a, labeled corr);

Figure 2 shows that these corrections all provide similar results if applied to the HE sample FWHM $\mathrm{H} \beta$. Error bars of Fig. 2 were estimated propagating the uncertainty values reported in Paper I for the full profiles, and those derived from specfit for the line components (assuming a minimum error of $10 \%$ ).

The middle panels of Fig. 2 show the ratios of corrected FWHM measurements as a function of the FWHM of the full $\mathrm{H} \beta$ profile. The low $\chi_{v}^{2}$ indicates that the $\chi_{v}^{2}$ associated with the ratios between $B C$ and symm, and BC and st is not significantly different from unity. In the case of $B C$ and corr the two measurements are different but only at $1 \sigma$ confidence level. The $F$ tests do not exclude that $\mathrm{BC}$, symmetrization, st, and reverberation corrections can be equivalent at a confidence level of $2 \sigma$. The bottom panel of Fig. 2 shows the behavior of the full and corrected FWHM versus the "symmetrized" $\mathrm{H} \beta$ FWHM. We consider the symmetrization, as it is relatively easy to apply (once the quasar rest frame is known), and the st correction (that does not even require the knowledge of the rest frame) as reference corrections. We reiterate the fact that these corrections are relatively minor but still significant: a $20 \%$ correction translates into a factor 1.44 correction in $M_{\mathrm{BH}}$. They do not undermine the value of the full line width of $\mathrm{H} \beta$ as a useful VBE (with the caveats discussed in Sect. 5.2), since the $\mathrm{H} \beta$ full line width remains preferable to the uncorrected Civ $\lambda 1549$ width for most objects.

\subsection{CIVג1549 in the full HE+FOS sample}

The results on the HE CIV $\lambda 1549$ profiles do not bode well for the use of Civ $\lambda 1549$ FWHM as a VBE, as also found by Sulentic et al. (2007) and other workers (Sect. 5.1 for a brief critical review). The presence of very large blueshifts in both Pop. A and B makes the situation even more critical than at low L. Figure 3 (top panel) shows that there is no obvious relation between the FWHM of Civ $\lambda 1549$ and the FWHM of $\mathrm{H} \beta$ if FOS + HE data are considered together.

For the Pop. A sources in the HE+FOS sample, Civ $\lambda 1549$ is broader than $\mathrm{H} \beta$, apart from in two cases in the HE sample, and FWHM(CIv 11549$)$ shows a broad range of values for similar FWHM $\mathrm{H} \beta$, that is, FWHM(CIv 21549$)$ is almost degenerate 

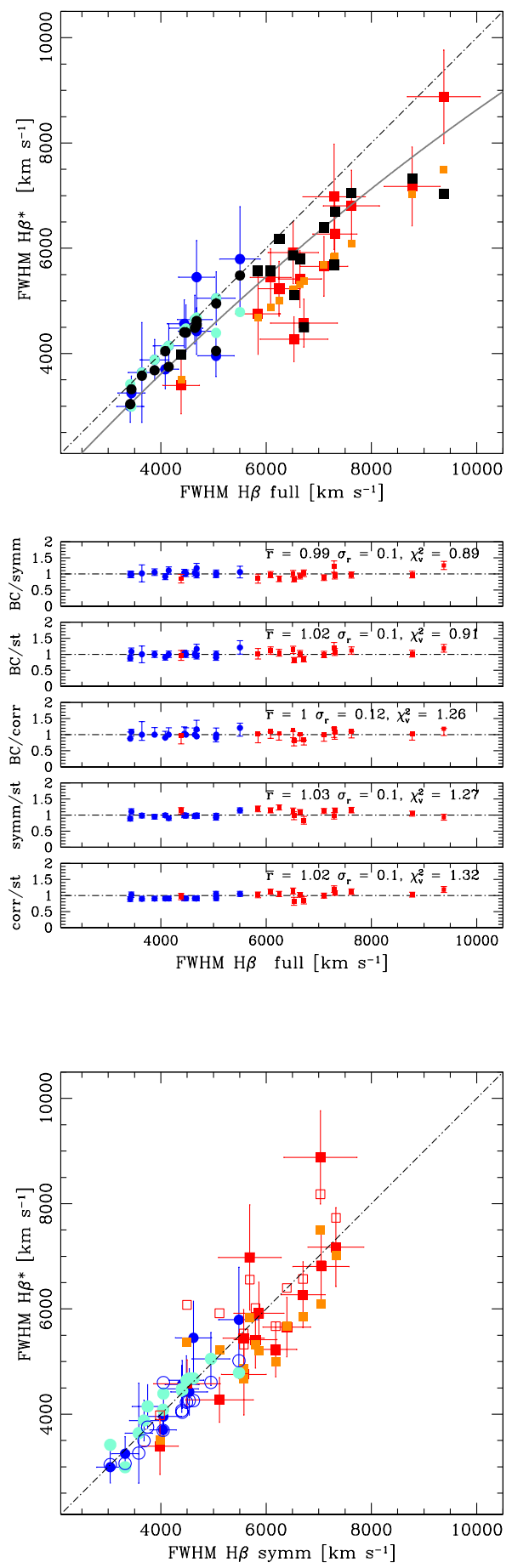

Fig. 2. Virial broadening estimators based on $\mathrm{H} \beta$, with several corrections applied to the HE sample. Top square panel: FWHM $\mathrm{H} \beta_{\mathrm{BC}}$ (blue (Pop. A) and red (Pop. B)) with error bars, symmetrized FWHM $\mathrm{H} \beta$ (symm, black), and FWHM $\mathrm{H} \beta$ corrected according to spectral type (st, aquamarine and dark orange for Pop. A and B respectively) versus FWHM of the full $\mathrm{H} \beta$ profile. The gray line traces the correction (corr) following the relation of Sulentic et al. (2006a) reported in Sect. 4. Middle panels: ratios of FWHM after various corrections vs. full profile FWHM. Panels from top show: BC/symm, BC/st, BC/corr, symm/ st, corr/st. Average values, standard deviation and normalized $\chi_{v}^{2}$ are reported in the upper-right corner of the panels. Bottom square panel: $\mathrm{BC}$, spectral type st and reverberation corrected (corr, open symbols) FWHM values vs. symmetrized FWHM.

with respect to $\mathrm{H} \beta$. The Civ $\lambda 1549$ line FWHM values are so much larger than the ones of $\mathrm{H} \beta$, thus making it possible that the
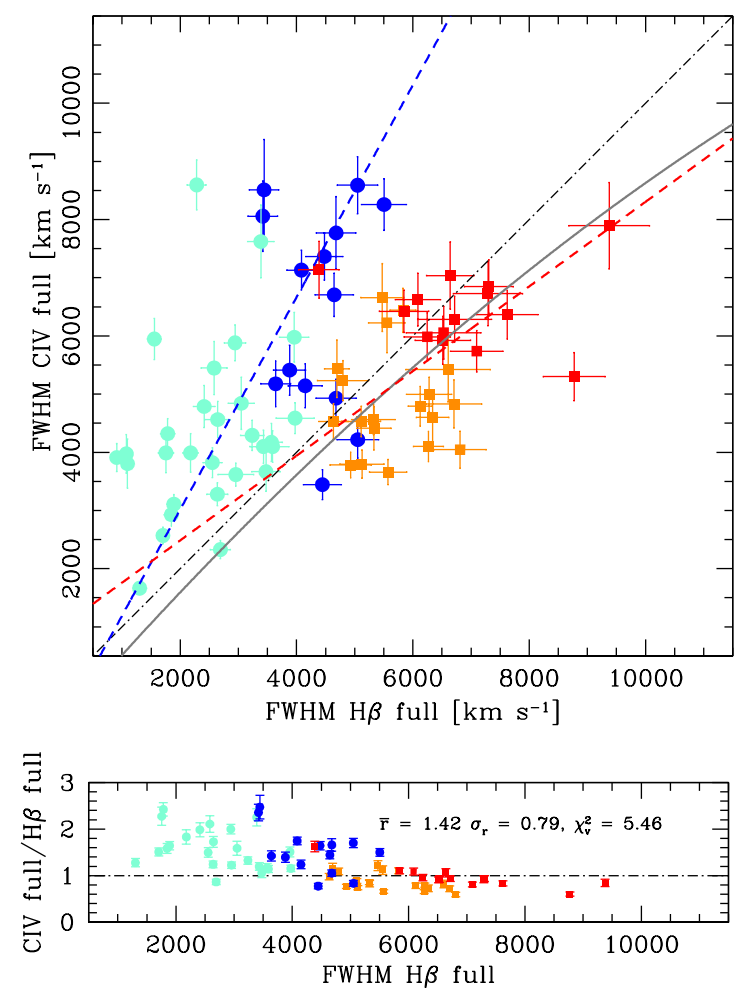

Fig. 3. Top panel: $\mathrm{FWHM}(\mathrm{Civ} \lambda 1549)$ vs. $\mathrm{FWHM}(\mathrm{H} \beta)$ (full profiles) for the FOS $+\mathrm{HE}$ sample. Data points are color-coded according to sample and population: HE Pop. A - blue circles $(\bullet)$, HE Pop. B - red squares (๘), FOS Pop. A - aquamarine circles (॰), and FOS Pop. B - golden squares ( $\square$ ). Best fitting least-square lines (dashed) are shown in blue for all Pop. A and red for all Pop. B. The black dot-dashed line is the equality line. The continuous gray line is the expected FWHM following the correction of Sulentic et al. (2006a, corr). Lower panel: ratio between FWHM CIv $\lambda 1549$ and FWHM $\mathrm{H} \beta$ as a function of FWHM $(\mathrm{H} \beta)$.

$M_{\mathrm{BH}}$ derived from FWHM Civ $\lambda 1549$ might be higher by even more than one order of magnitude. Formally, the Pearson's correlation coefficient $r \approx 0.52$ is highly significant for a sample of $n=43$, with significance at a confidence level $\approx 4.5 \sigma$. A weighted least-square fit yields $F W H M($ CIV $\lambda 1549)=(1.822 \pm$ $0.204) F W H M(\mathrm{H} \beta)+(-624 \pm 677) \mathrm{km} \mathrm{s}^{-1}$, with a significant scatter, rms $\approx 1959 \mathrm{~km} \mathrm{~s}^{-1}$. Unfortunately it is not possible to apply a simple Civ $\lambda 1549$ symmetrization as done for $\mathrm{H} \beta$ : subtracting $2 \cdot c\left(\frac{1}{2}\right)$ from FWHM CIV $\lambda 1549$ leads to corrections that are unrealistically large.

If we combine the Pop. B FOS and HE samples, FWHM Civ $\lambda 1549$ and $\mathrm{H} \beta$ become loosely correlated (the Pearson's correlation coefficient is $\approx 0.4$, significant at $P \approx 98 \%$ for a sample of 33 objects). A weighted least-square fit yields $F W H M(\operatorname{Civ} \lambda 1549) \approx$ $(0.764 \pm 0.165) F W H M(\mathrm{H} \beta)+(810 \pm 1030) \mathrm{km} \mathrm{s}^{-1}$, and $\mathrm{rms} \approx$ $1090 \mathrm{~km} \mathrm{~s}^{-1}$, with a significant deviation from the 1:1 relation. In the case of Pop. B sources, the trend implies FWHM CIv $\lambda 1549$ FWHM $\mathrm{H} \beta$, and even a slightly narrower FWHM CIv $\lambda 1549$ with respect to $\mathrm{H} \beta$.

The large scatter induced by using an uncorrected Civ $\lambda 1549$ line FWHM may have contributed to the conclusion that line width does not contribute significantly to $M_{\mathrm{BH}}$ determinations (Croom 2011).

\subsection{Practical usability of CIv $11549_{B C}$}

The fitting procedure scaled the $\mathrm{H} \beta$ profile to model the red side of CIv $\lambda 1549$ meaning that the FWHM CIv $\lambda 1549_{\mathrm{BC}}$ estimate 
is not independent from FWHM $\mathrm{H} \beta_{\mathrm{BC}}$. The FWHM values of the two BCs are in agreement because of this enforced condition.

The Civ $\lambda 1549_{\mathrm{BC}}$ extraction is very sensitive to the assumed rest frame, and also requires that the CIV $\lambda 1549$ line be cleaned from contaminants such as FeII (weak) and HeII $\lambda 1640$ (moderate, but flat-topped and gently merging with the Civ $\lambda 1549$ red wing; Marziani et al. 2010; Fine et al. 2010; Sun et al. 2018). Without performing a line profile decomposition, one can consider the width of the red side with respect to rest frame as the half width at half maximum (HWHM) of the virial component. Again this requires (1) an accurate redshift that can be set, in the context of high-redshift quasars, either by using the $\mathrm{H} \beta$ narrow component or by the [OII] $\lambda 3727$ doublet (Eracleous \& Halpern 2004; Hu et al. 2008), and (2) the decomposition from HeII $\lambda 1640$ emission blended on the CIV $\lambda 1549$ red side. If [OII] $\lambda 3727$ is covered, then $\mathrm{MgII} \lambda 2800$ is also likely to be covered. As mentioned in Sect. 3, the MgII $\lambda 2800$ line width is a reliable VBE for the vast majority of type-1 AGNs. The same is not true for CIV $\lambda 1549$. For spectra where CIV $\lambda 1549$ is conveniently placed at $z \gtrsim 1.45$, the [OII] $\lambda 3727$ line is shifted beyond $9000 \AA$, a domain where intense sky emission makes it difficult to analyze a relatively faint narrow line. The extraction of Civ $\lambda 1549_{\mathrm{BC}}$ is therefore not a viable solution if single-epoch Civ $\lambda 1549$ observations are available without the support of at least a narrow LIL that may set a reliable rest frame. This is unlikely to occur on the same optical spectra. An alternative strategy for $M_{\mathrm{BH}}$ estimation using Civ $\lambda 1549$ FWHM should consider the origin of the Civ $\lambda 1549$ nonvirial broadening.

\subsection{Reducing CIvג1549 to a VBE estimator consistent with $H \beta$}

The main results of Paper I suggest a strong dependence of the CIV $\lambda 1549$ blueshift on $L / L_{\text {Edd }}$, especially above a threshold value $L / L_{\mathrm{Edd}} \approx 0.2 \pm 0.1$ (Sulentic et al. 2014, and references therein). A correlation between Eddington ratio and the FWHM(CIv $\lambda 1549)$ to $\mathrm{FWHM}(\mathrm{H} \beta)$ ratio (i.e., $1 / \xi_{\text {CIV }}$, cf., Saito et al. 2016) is detected at a high significance level (Pearson's correlation coefficient $r \approx 0.55$ ) joining all FOS RQ sources of Sulentic et al. (2007; Fig. 4). In this context, $L / L_{\text {Edd }}$ was computed from the $M_{\mathrm{BH}}$ scaling law of Vestergaard \& Peterson (2006) using the FWHM of $\mathrm{H} \beta$ and $\lambda L_{\lambda}(5100)$. A bisector best fit with SLOPES (Feigelson \& Babu 1992) yields

$\log \frac{1}{\xi_{\mathrm{CIV}}} \approx(0.426 \pm 0.043) \frac{L}{L_{\mathrm{Edd}}}+(0.401 \pm 0.035)$.

An $L / L_{\text {Edd }}$-dependent correction is in principle a valid approach. However, how to calculate $L / L_{\text {Edd }}$ from UV spectra without resorting to $\mathrm{H} \beta$ observations is not obvious. In addition, FWHM $\mathrm{H} \beta$ is strongly affected by orientation and yields biased values of $L / L_{\text {Edd }}$ (Sect. 5.2.2). Both $\operatorname{FWHM}\left(\mathrm{H} \beta_{\mathrm{BC}}\right) / \mathrm{FWHM}(\mathrm{CIV} \lambda 1549)$ and $c\left(\frac{1}{2}\right)$ are correlated with Eddington ratio. Consistently, the CIV $\lambda 1549$ blueshift is correlated with FWHM Civ $\lambda 1549$ (Paper I, Coatman et al. 2016), and accounts for the broadening excess in the Civ $\lambda 1549$ FWHM. Measurements of the Civ $\lambda 1549$ blueshift or the $\mathrm{FWHM}\left(\mathrm{H} \beta_{\mathrm{BC}}\right) / \mathrm{FWHM}(\mathrm{Crv} \lambda 1549)$ can be used as proxies for $L / L_{\text {Edd }}$. At the same time, Paper I reveals a weaker correlation with $L$, which is expected in the case of a radiation-driven wind. If the correction factor is $\xi_{\mathrm{CIV}}=F W H M\left(\mathrm{H} \beta_{\mathrm{BC}}\right) / F W H M(\mathrm{CIV} \lambda 1549)$, then it should include a term in the form $1 / \zeta\left(L, L / L_{\mathrm{Edd}}\right)$.

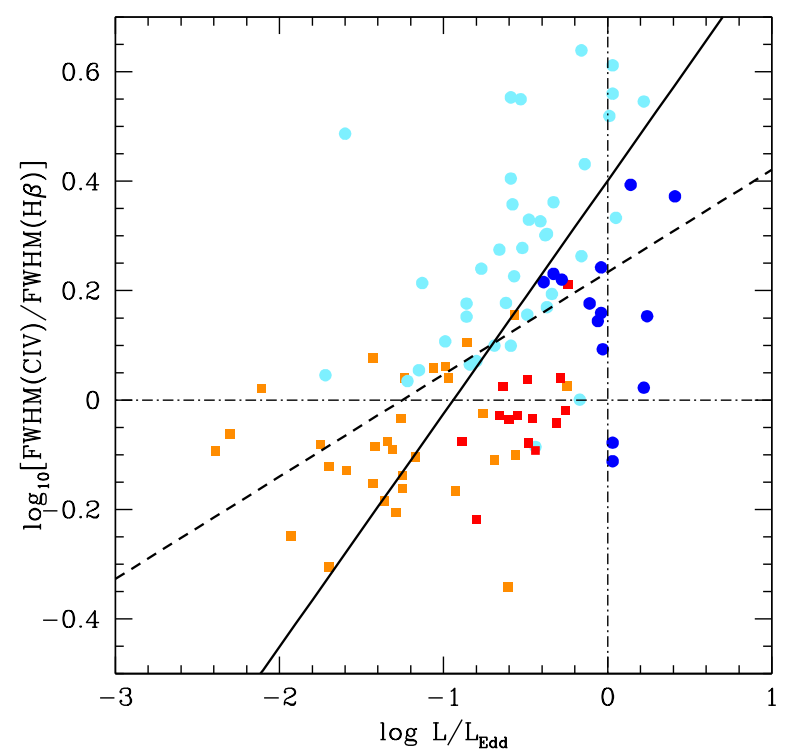

Fig. 4. Relation between the logarithm of the FWHM ratio of Civ $\lambda 1549$ to $\mathrm{H} \beta$ and the logarithm of the Eddington ratio $L / L_{\mathrm{Edd}}$. The vertical dot-dashed line traces the Eddington limit. The colors and shapes of symbols are as in Fig. 3. The dashed line is an unweighted least-squares fit, and the filled line was obtained with the bisector method (Feigelson \& Babu 1992).

\subsection{Calibrating empirical corrections on FWHM CIVג1549}

Coatman et al. (2017) introduced a nonparametric measurement of the Civ $\lambda 1549$ blueshift associated with the wavelength that splits the line flux in equal parts on its blue and red sides (flux bisector). The flux bisector is strongly correlated with $c\left(\frac{1}{2}\right)$ and $c\left(\frac{1}{4}\right)$, and $c\left(\frac{1}{2}\right)$ and $c\left(\frac{1}{4}\right)$ are correlated among themselves in the FOS+HE sample (Pearson's $r \approx 0.95): c\left(\frac{1}{2}\right)=(0.773 \pm$ $0.307) c\left(\frac{1}{4}\right)-(58 \pm 65) \mathrm{km} \mathrm{s}^{-1}$. The bisector correlation is stronger with $c\left(\frac{1}{4}\right)$ (Pearson's correlation coefficient $r \approx 0.98$ ), with flux bisector $\sim(0.98 \pm 0.04) c\left(\frac{1}{4}\right)+(220 \pm 110) \mathrm{km} \mathrm{s}^{-1}$ (Fig. 5). The lower panel of Fig. 5 shows a few objects where the difference between the $c\left(\frac{1}{4}\right)$ and the flux bisector is $\gtrsim 20 \%$; these sources are either with small shifts (within the measurement uncertainties; shaded area of Fig. 5), or sources strongly affected by broad absorptions, for which measurement of blueshift is tricky regardless of the method employed. Therefore, it is possible to apply Eq. (4) of Coatman et al. (2017) substituting the $c\left(\frac{1}{4}\right)$ to the fluxbisector blueshift measurements:

$\xi_{\mathrm{CIV}, 0}=\frac{1}{a\left(-\frac{c\left(\frac{1}{4}\right)}{1000}\right)+b}$,

with $a=0.41 \pm 0.02$ and $b \approx 0.62 \pm 0.04$ (the minus sign is because Coatman et al. (2017) assumed blueshifts to be positive), to correct the FWHM CIV $\lambda 1549$ of the FOS+HE sample. The resulting trend is shown in Fig. 6. Equation (4) of Coatman et al. (2017) undercorrects both Pop. A and B sources at low $L$ (the FOS sample) and provides a slight overcorrection for the HE sources. The correction of Coatman et al. (2017) does not yield FWHM (CIV 21549) in agreement with the observed values of $\mathrm{FWHM}(\mathrm{H} \beta)$. This does not necessarily mean that the FWHM(CIv $\lambda 1549)$ values are incorrect, as $\operatorname{FWHM}(\mathrm{H} \beta)$ is likely more strongly affected by orientation effects than FWHM(CIV 11549$)$; see the discussion in Sect. 5.2. 


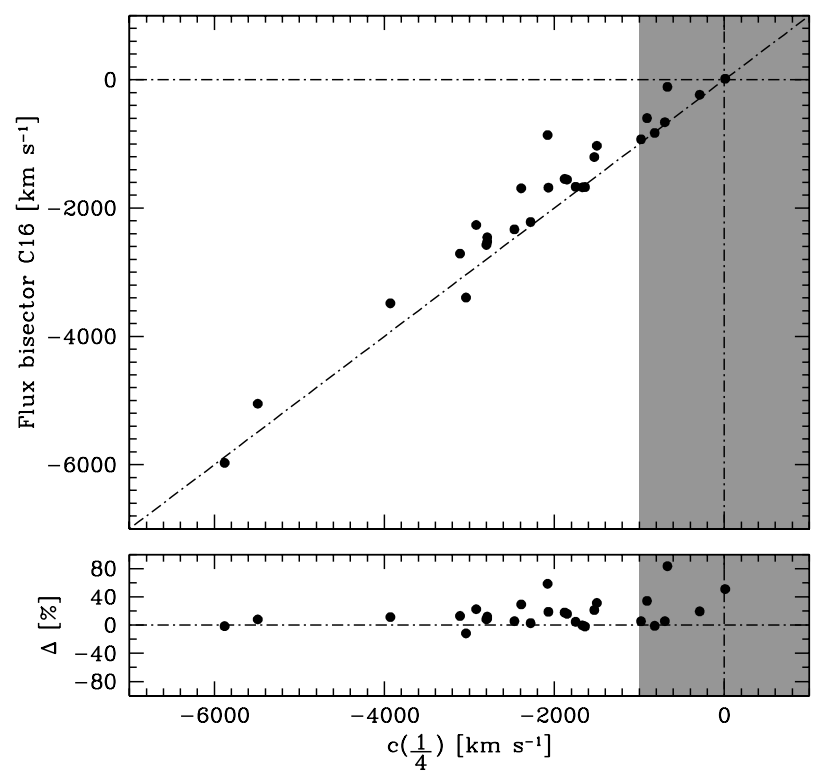

Fig. 5. Top panel: bisector flux estimator of Coatman et al. (2017) vs. $c\left(\frac{1}{4}\right)$, in $\mathrm{km} \mathrm{s}^{-1}$. The dot-dashed line is the equality line. Bottom panel: percentage residuals. The shaded areas indicate the average errors of measurements at a $2 \sigma$ confidence level computed from Table 4 of Paper I.

A correction dependent on luminosity reduces the systematic differences between the various samples in the present work but it has to be separately defined for Pop. A and B (Fig. 7). The following expression provides a suitable fitting law, with $a, b, c$ different for Pop. A and B.

$$
\xi_{\mathrm{CIV}, 1}=\frac{1}{b\left(a-\log \lambda L_{\lambda}(1450)\right) \cdot\left(\left|\frac{c\left(\frac{1}{2}\right)}{1000}\right|\right)+c} .
$$

Here we consider the $c\left(\frac{1}{2}\right)$ because of its immediate connection with the FWHM, and because it is highly correlated with $c\left(\frac{1}{4}\right)$ (Sect. 5.5). Equation (6) is empirical: it entails a term proportional to shift and one to the product of $\log L_{1450}$ and shift. Multivariate, nonlinear lsq results for Eq. (6) are reported in the first rows of Table 1. For Pop. A, the correction is rather similar to the one of Coatman et al. (2017), and is driven by the large blueshifts observed at high $L / L_{\text {Edd }}$. The luminosity-dependent factor accounts for low-luminosity sources that are not present in the Coatman et al. (2017) sample. The use of the absolute value operator provides an improvement with respect to the case in which blueshifts are left negative. There are only three objects for which $c\left(\frac{1}{2}\right)$ is positive. The improvement is understandable if one considers any Civ $\lambda 1549$ shift as affecting the difference between the FWHM of Civ $\lambda 1549$ and $\mathrm{H} \beta$. An A(+) sample was defined from the Pop. A sample minus three objects with positive $c\left(\frac{1}{2}\right)$, that is, all $\mathrm{A}(+)$ sample sources show blueshifts. No significant improvement was found with respect to the previous sample.

The correction for Pop. B is less well defined, considering the uncertainty in $a$, and the low value of $b$ (Table 1 ). The corrections for Pop. B still offer an improvement because they remove significant bias (as evident by comparing Figs. 3 and 7). In practice, for Pop. B, at low $L$ the $\xi_{\text {CIV }}$ could be considered constant to a zero-order approximation, with $\xi_{\mathrm{CIV}} \sim 1$. In other words, when the velocity field is predominantly virial, and no promi-
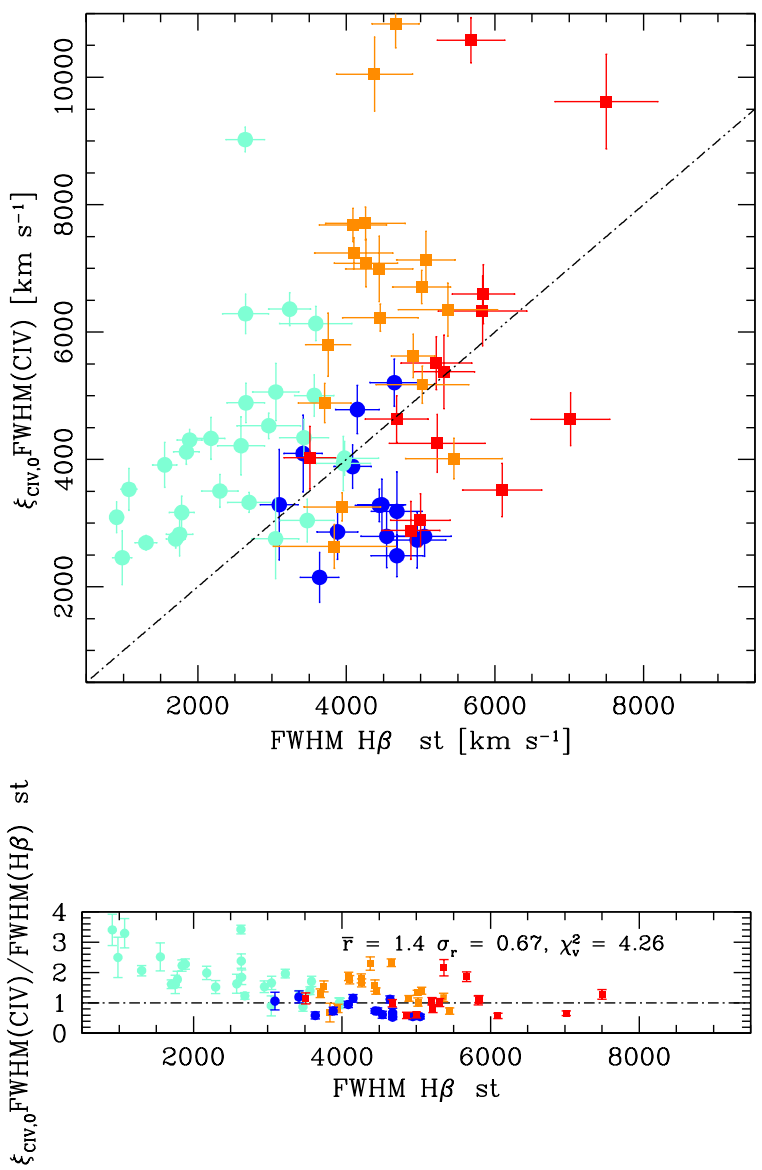

Fig. 6. Top panel: FWHM(CIv 11549$) \mathrm{C} 16$ (i.e., corrected following Coatman et al. 2016), vs. $\mathrm{FWHM}(\mathrm{H} \beta)$ st for the FOS+HE sample. Meaning of symbols is the same as for Fig. 3. The black dot-dashed line is the equality line. Bottom panel: residuals. Average ratio, dispersion, and $\chi_{v}^{2}$ refer to all sources.

nent blueshifted component affects the line width, CIv $\lambda 1549$ may be somewhat broader than $\mathrm{H} \beta$ as expected for the stratification revealed by reverberation mapping of lines from different ionic species (Peterson \& Wandel 1999, 2000). The fit is consistent with $\xi_{\mathrm{CIV}}$ depending on shift but only weakly on luminosity. The Pop. B correction is so ill-defined that larger samples are needed for a better determination of its coefficients.

Slightly different fitting laws

$$
\xi_{\mathrm{CIV}, 2}=\frac{1}{a+b \log \lambda L_{\lambda}(1450)+c \frac{c\left(\frac{1}{2}\right)}{1000}},
$$

which considers a linear combination $\log \lambda L_{\lambda}(1450)$ and shift, and

$\xi_{\mathrm{CIV}, 3}=\frac{1}{a-b \log \lambda L_{\lambda}(1450) \cdot \frac{c\left(\frac{1}{2}\right)}{1000}}$,

which assumes a dependence from the product $\log \lambda L_{\lambda}(1450)$ and shift, provide consistent results, with fitting parameters, $1 \sigma$ confidence level associated uncertainty, and root mean square (rms) residuals of $\xi_{\text {CIV }}$ reported in Table 1 . The fitting relations yield a lower residual scatter in $\xi_{\mathrm{CIV}}$ than assuming no luminosity dependence. For instance, using Eq. (7) we obtain a scatter in $\xi_{\mathrm{CIV}, 2}$ that is a factor 1.86 lower than if Eq. (5) is used. We also considered the AI in place of $c\left(\frac{1}{2}\right)$ in Eq. (6) (without the absolute value operator; bottom rows of Table 1). The AI has a 
Table 1. Fits of $\xi_{\text {CIV }}$.

\begin{tabular}{|c|c|c|c|c|c|}
\hline Sample & $a \pm \delta a$ & $b \pm \delta b$ & $c \pm \delta c$ & $\mathrm{rms}_{\xi}$ & d.o.f. \\
\hline \multicolumn{6}{|c|}{$\xi_{\mathrm{CIV}, 1} \approx 1 /(b *(a-x) *|y|+c)$} \\
\hline A & -0.30930 .1581 & 0.34340 .0881 & 1.07630 .0949 & 0.198 & 40 \\
\hline $\mathrm{B}$ & 3.922414 .1170 & 0.02060 .0568 & 0.98450 .0602 & 0.187 & 30 \\
\hline $\mathrm{A}+\mathrm{B}$ & -0.18050 .2603 & 0.19780 .0584 & 1.01170 .0641 & 0.227 & 73 \\
\hline \multicolumn{6}{|c|}{$\xi_{\mathrm{CIV}, 1} \approx 1 /(b *(a-x) * y+c)$} \\
\hline A & -0.41610 .3325 & -0.18250 .0693 & 1.32450 .0919 & 0.222 & 40 \\
\hline $\mathrm{B}$ & 28.967496 .591 & -0.00300 .0486 & 1.01820 .0449 & 0.187 & 30 \\
\hline $\mathrm{A}+\mathrm{B}$ & -0.13460 .4991 & -0.12020 .0467 & 1.15080 .0510 & 0.238 & 73 \\
\hline \multicolumn{6}{|c|}{$\xi_{\mathrm{CIV}, 2} \approx 1 /(a+b * x+c * y)$} \\
\hline A & 0.3560 .160 & -0.34800 .0580 & -0.3510 .0814 & 0.199 & 40 \\
\hline B & 1.0800 .126 & 0.02230 .0416 & -0.07640 .0515 & 0.186 & 30 \\
\hline $\mathrm{A}+\mathrm{B}$ & 0.76050 .125 & -0.14600 .0452 & -0.22040 .0532 & 0.237 & 73 \\
\hline \multicolumn{6}{|c|}{$\xi_{\mathrm{CIV}, 3} \approx 1 /(a-b * x * y)$} \\
\hline A & 1.30360 .0884 & -0.14080 .0481 & & 0.222 & 41 \\
\hline B & 1.03430 .0436 & -0.04630 .0255 & & 0.187 & 31 \\
\hline $\mathrm{A}+\mathrm{B}$ & 1.14700 .0488 & -0.11160 .0288 & $\ldots$ & 0.237 & 74 \\
\hline \multicolumn{6}{|c|}{$\xi_{\mathrm{CIV}, \mathrm{AI}} \approx 1 /(b *(a-x) * z+c)$} \\
\hline A & -1.70040 .5528 & -1.05750 .4703 & 1.55150 .1183 & 0.240 & 40 \\
\hline $\mathrm{B}$ & -2.3460 .3153 & 0.75710 .3026 & 1.10570 .0413 & 0.184 & 30 \\
\hline $\mathrm{A}+\mathrm{B}$ & 5.14721 .138 & -0.11210 .3292 & 1.24740 .0501 & 0.257 & 73 \\
\hline
\end{tabular}

Notes. $x=\log \lambda L_{\lambda}(1450)-48$, for $\log \lambda L_{\lambda}(1450) \lesssim 47.5\left[\mathrm{ergs}^{-1}\right] ; y=c\left(\frac{1}{2}\right) / 1000, z=\mathrm{AI}$.

non-negligible advantage to be independent from the choice of the rest frame. The AI is correlated with both $c\left(\frac{1}{4}\right)$ and $c\left(\frac{1}{2}\right)$, and shows higher correlation with $c\left(\frac{1}{4}\right)$ (Pearson's $r \approx 0.66$ ). However, the scatter in $\xi_{\text {CIV ,A.I. }}$ is unfortunately large, and would imply a scatter $\approx 1.5$ higher in $M_{\mathrm{BH}}$ estimates than in the case where Eq. (6) is considered for Pop. A.

If Pop. A and B are considered together, the final scatter in $\xi_{\mathrm{CIV}}$ is close for the different fitting function (Eq. (6) yields a slightly better result) but much higher than if Pop. A and B are kept separated. It is therefore necessary to distinguish between Pop. A and B as the intrinsic structure of their BLR may be different (e.g., Goad \& Korista 2014; Wang et al. 2014a). In Pop. B, at low $L / L_{\mathrm{Edd}}$, the lines are mainly broadened following a virial velocity field (Peterson \& Wandel 2000). The relative prominence of the blueshifted to the virialized component (ratio BLUE over BC) is lower in Pop. B than in Pop. A, a consequence of the low $L / L_{\text {Edd }}$ for Pop. B sources. Both properties are expected to contribute to the overall consistency between $\mathrm{H} \beta$ and CIv $\lambda 1549$ profiles in Pop. B sources. At any rate, $\xi_{\text {CIV }}$ should always be $\lesssim 1$, with $\xi_{\mathrm{CIV}} \approx 1$ for Pop. B at low $L$, and $\xi_{\mathrm{CIV}} \ll 1$ in case of very large shifts, as in Pop. A at high $L$.

It is possible in most cases to distinguish between Pop. A and B from the UV spectrum emission blend, making the correction applicable at least to a fraction of all quasars in large samples. Several criteria were laid out by Negrete et al. (2014): (1) broad line width; (2) evidence of a prominent red wing indicative of a VBC; and (3) prominence of CIII] $\lambda 1909$. Population B sources show a Civ $\lambda 1549$ red wing and strong CIII] $\lambda 1909$ in the $1900 \AA$. Extreme Pop. A (xA) sources are easy to recognize; they show strong AlIII $\lambda 1860$ in $1900 \AA$ blend and low $W$ (CIv 11549). A prototypical composite spectrum of xA sources is shown by Martínez-Aldama et al. (2018). However, some intermediate cases along the MS (i.e., spectral type A1) may be easier to misclassify. Also, with only the UV spectral range available, the redshift estimate may be subject to large errors.
Negrete et al. (2014) provide a helpful recipe; however, their recipe applied to three of their eight sources allowed for a precision of $\sim 100 \mathrm{~km} \mathrm{~s}^{-1}$ in the rest frame, but the remaining five had an average uncertainty $\gtrsim 500 \mathrm{~km} \mathrm{~s}^{-1}$.

\section{Discussion}

Recently, the problems outlined in earlier works by Sulentic et al. (2007) and Netzer et al. (2007) have been ascribed to a "bias" in the Civ $\lambda 1549 M_{\mathrm{BH}}$ estimates (Denney et al. 2016). The CIv $\lambda 1549 M_{\mathrm{BH}}$ bias is dependent on the location in the 4DE1 quasar MS: Figs. 2 and 3 clearly show the different behavior for Populations $\mathrm{A}$ and B. By the same token, an $L / L_{\text {Edd }}$-dependent correction is in principle a valid approach, as $L / L_{\text {Edd }}$ is probably one of the main drivers of the MS (Boroson \& Green 1992; Sulentic et al. 2000a; Sun \& Shen 2015). Unfortunately, several recent works still ignore 4DE1-related effects (or, in other words, MS trends). For instance, scaling laws derived from the pairing of the virial products for all sources with reverberation mapping data should be viewed with care (as shown by the reverberation mapping results of Du et al. 2018).

\subsection{CIVג1549 and $H \beta$ as $M_{B H}$ estimators: input from recent works}

Attempts at using the Crv $\lambda 1549$ as a VBE have been renewed in the last few years, not least because CIV $\lambda 1549$ can be observed in the optical and NIR spectral ranges over which high-redshift quasars have been discovered and are expected to be discovered in the near future. The large Civ $\lambda 1549$ blueshifts indicate that part of the BLR gas is under dynamical conditions that are far from virialized equilibrium. At high Eddington ratios, ionized gas may escape from the galactic bulge, and even be dispersed into the intergalactic medium, as predicted by numerical simulations (e.g., Debuhr et al. 2012), and at high luminosity 

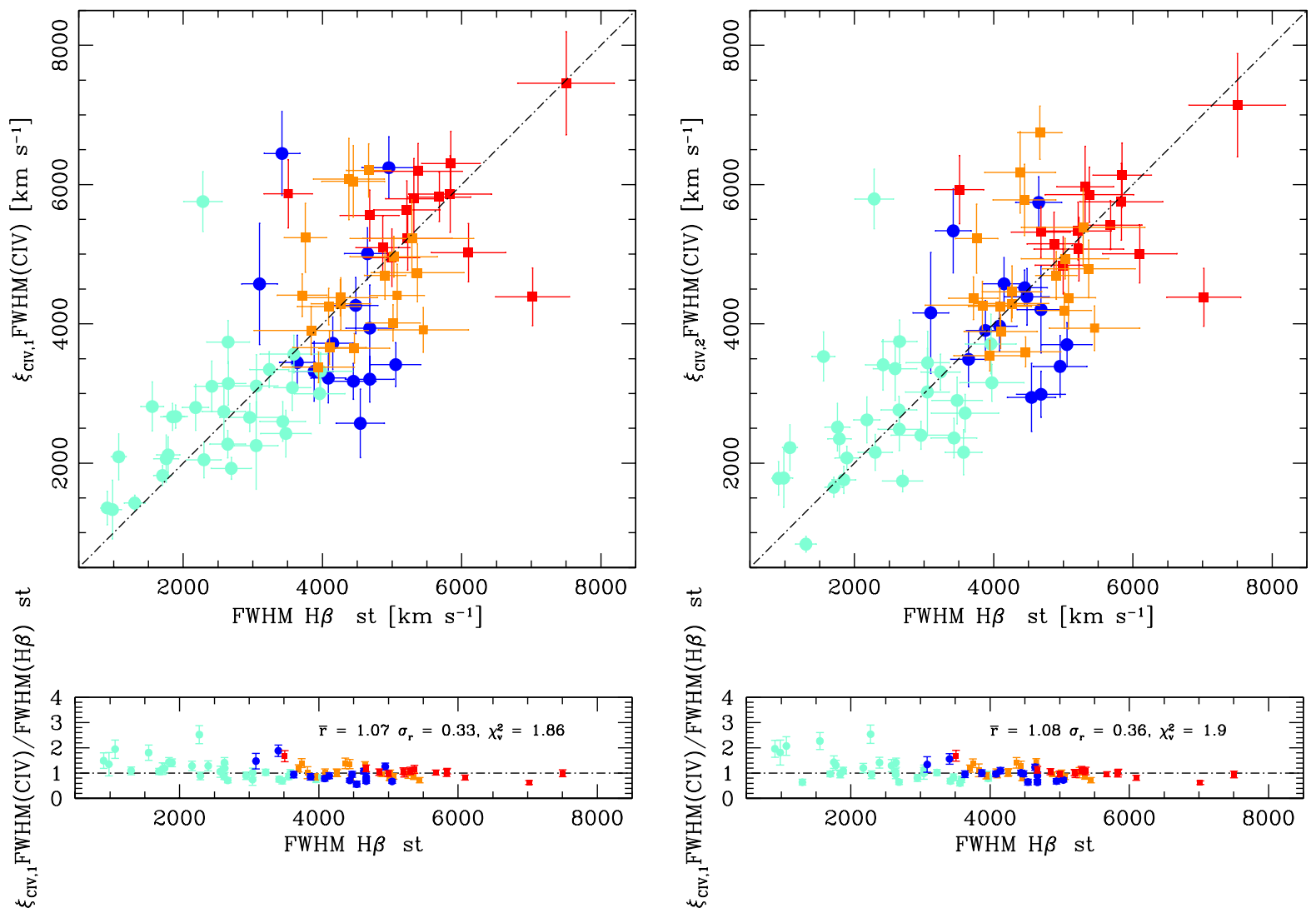

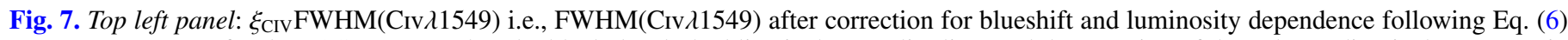
vs. $\mathrm{FWHM}(\mathrm{H} \beta) \mathrm{cm}$ for the FOS+HE sample. The black dot-dashed line is the equality line, and the meaning of the color-coding is the same as in Fig. 3. Top right: as in top panel but with $\xi_{\text {CIV }}$ computed from Eq. (7).

$\left(\log L \gtrsim 47\left[\mathrm{erg} \mathrm{s}^{-1}\right]\right)$ they might have a significant feedback effect on the host galaxy (Marziani et al. 2016).

A firm premise is that the disagreement between $\mathrm{H} \beta$ and Civ $\lambda 1549$ mass estimates is not a matter of S/N (Denney et al. 2013). The CIV $\lambda 1549$ line width suffers from systematic effects which emerge more dramatically at high $\mathrm{S} / \mathrm{N}$, i.e., when it is possible to appreciate the complexity of the Civ $\lambda 1549$ profile. Given this basic result, recent literature can be tentatively grouped into three main strands: (1) low-redshift studies involving FOS and Cosmic Origin Spectrograph (COS) spectra to cover CIv $\lambda 1549$; (2) high-redshift studies, where the prevalence of large Civ $\lambda 1549$ shifts is high; and (3) studies attempting to correct the Civ $\lambda 1549$ FWHM and reduce it to an equivalent of $\mathrm{H} \beta$, some of them employing results that are directly connected to the MS contextualization of quasar properties.

Low-redshift studies. A large systematic analysis of the Civ $\lambda 1549$ profiles paired to $\mathrm{H} \beta$ emission was carried out using HST/FOS (in part used for the present work) and optical observations (Sulentic et al. 2007). The results of this study emphasized the role of the CIv $\lambda 1549$ line width in the $M_{\mathrm{BH}}$ estimates. Figure 6 of Sulentic et al. (2007) clearly shows the importance of placing sources in an E1 context: estimates of the masses could be easily overestimated by a factor $\lesssim 100$ for extreme Pop. A sources such as I Zw 1, while for Pop. B, CIv $\lambda 1549$ and $\mathrm{H} \beta M_{\mathrm{BH}}$ estimates appeared more consistent albeit with a large scatter. The line width (be it the FWHM or the velocity dispersion $\sigma$ ) remains a major factor in CIv $\lambda 1549$ versus $\mathrm{H} \beta M_{\mathrm{BH}}$ determinations since broadening enters squared in the scaling laws (Kelly $\&$ Bechtold 2007). Similar warnings on using Civ $\lambda 1549$ FWHM were issued by Netzer et al. (2007). Low-redshift samples are less affected by the Eddington ratio bias that is cutting lowEddington ratio sources at a given $M_{\mathrm{BH}}$ for a fixed flux limit (Sulentic et al. 2014). Therefore, it may not be surprising to find studies based on excellent spectra that find an overall consistency between $\mathrm{H} \beta$ and $\mathrm{CIV} \lambda 1549 M_{\mathrm{BH}}$ estimates. Intrinsic scatter is probably high if full line widths without corrections are used: Tilton \& Shull (2013) find $\approx 0.5 \mathrm{dex}$ from COS observations of low-redshift quasars. Denney et al. (2013) claim to be able to reduce the disagreement between $M_{\mathrm{BH}}$ derived using $\mathrm{H} \beta$ and CIv $\lambda 1549$ to $\approx 0.24$ dex by using the velocity dispersion of the Civ $\lambda 1549$ line. Since the Civ $\lambda 1549$ profile in the Denney et al. (2013) sample almost never shows large blueshifts, which may be associated to a velocity shear in outflowing gas, these results appear consistent with the Pop. B properties of the FOS sample.

High-redshift studies. These studies generally agree on the fact that the Civ 21549 FWHM is poorly correlated with the Balmer line FWHM. Shen \& Liu (2012) describe the scatter between CIv $\lambda 1549$ and $\mathrm{H} \beta \mathrm{FWHM}$ as due to an irreducible part $(\approx 0.12$ dex $)$, and a part that correlates with the blueshift of the Civ $\lambda 1549$ centroid relative to that of $\mathrm{H} \beta$. These latter authors propose scaling laws in which the virial assumption is abandoned, that is, with the exponent of the line FWHM being significantly different from two. For Civ $\lambda 1549$, this means correcting for the overbroadening associated with the nonvirial component. The scaling law introduced by Park et al. (2013) is consistent with the approach by Shen \& Liu (2012) and implies $M_{\mathrm{BH}} \propto F W H M^{0.5}$, that is, a FWHM dependence that is very different from the one expected from a virial law $\left(M_{\mathrm{BH}} \propto\right.$ $\left.F W H M^{2}\right)$. As shown in Fig. 8, the scaling law suggested by Park et al. (2013) applied to the HE sample properly corrects for the 

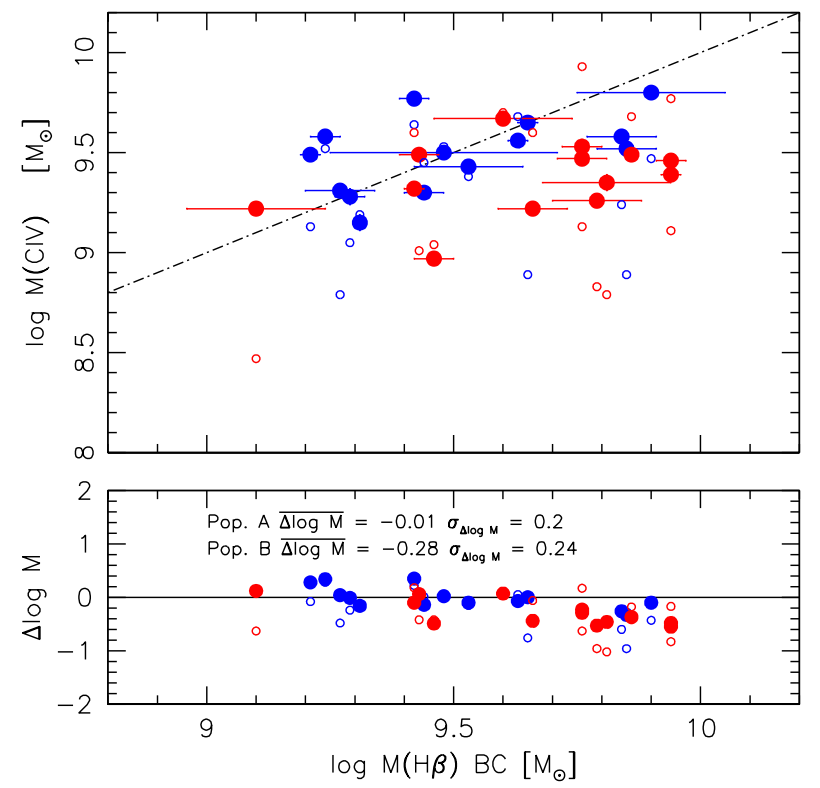

Fig. 8. Black hole mass computed from the fiducial relation of Vestergaard \& Peterson (2006) based on FWHM H $\beta$ vs. the ones computed from the Civ $\lambda 1549$ FWHM following Park et al. (2013), for Pop. A (blue) and B (red) HE sources. Values of $M_{\mathrm{BH}}$ obtained from the Vestergaard \& Peterson (2006) Civ $\lambda 1549$ scaling law after the correction suggested by Brotherton et al. (2015, small dots) are shown by small open circles. Lower panel: residuals as a function of $M_{\mathrm{BH}}$. The average and the scatter reported for Populations A and B refer to the Park et al. (2013) scaling laws.

overbroadening of Pop. A sources, but overcorrects the width of Pop. B, yielding a large deviation from the $\mathrm{H} \beta$-derived $M_{\mathrm{BH}}$ values (on average $\approx 0.28 \mathrm{dex}$ ).

Studies exploiting MS trends. The results reported in Sect. 4 and in Paper I indicate that any solution seeking to bring Civ $\lambda 1549$ $M_{\mathrm{BH}}$ estimates into agreement with the ones from $\mathrm{H} \beta$ cannot exclude the strong $L / L_{\text {Edd }}$ dependence of the CIV $\lambda 1549$ blueshift that is in turn affecting the Civ $\lambda 1549$ FWHM (Fig. 4). The discussion in Sects. 3 and 4.5 here identifies the Civ $\lambda 1549$ blueshift as an expedient $L / L_{\text {Edd }}$ proxy. Any parameterization of the blueshifted amplitude such as $c\left(\frac{1}{2}\right)$, the flux bisector of Coatman et al. (2016), or the ratio FWHM(Civ $\lambda 1549) /$ $\operatorname{FWHM}(\mathrm{H} \beta)$ takes into account the MS trends in Civ $\lambda 1549$ properties due to the $L / L_{\text {Edd }}$ and Civ $\lambda 1549$ blueshift correlation. Another $L / L_{\text {Edd }}$ proxy may involve the Sirv $\lambda 1397 /$ Civ $\lambda 1549$ peak ratio: at low Sirv $\lambda 1397 / \operatorname{Crv} \lambda 1549$ the $M_{\mathrm{BH}}$ is underestimated with respect to $\mathrm{H} \beta$, and at high Sirv $\lambda 1397 / \operatorname{Crv} \lambda 1549$ the mass is overestimated (Brotherton et al. 2015). Since the ratio Sirv $\lambda 1397 /$ Crv $\lambda 1549$ is a known 4DE1 correlate (Wills et al. 1993; Bachev et al. 2004), these results confirm that FWHM Civ $\lambda 1549$ leads to an overestimate of $M_{\mathrm{BH}}$ for Pop. A (as Pop. A outflows produce blueshifted emission that significantly broadens the line (Fig. 3; cf. Denney et al. 2012). In our sample, however, applying the correction suggested by Brotherton et al. (2015), that is, $\delta \log M \approx-1.23 \log \frac{1400}{\text { CIV }}-0.91$, to the masses derived from the Park et al. (2013) would move the $M_{\mathrm{BH}}$ of Pop. B further down, leading to a further increase of the overcorrection, and also destroying the agreement for Pop. A sources; on top of the $\propto F W H M^{0.5}$ law, the additional correction is $\delta \log M \approx-0.91$ if $\frac{1400}{\mathrm{CIV}} \sim 1$. The correction is lower but still negative for most Pop. B sources where $\frac{1400}{\text { CIV }} \sim 0.3$, exacerbating the disagreement between the $\mathrm{H} \beta$ and $\mathrm{C}$ Iv $\lambda 1549$ derived masses. Better consistency is achieved if the correction of
Brotherton et al. (2015) is applied to the Vestergaard \& Peterson (2006) scaling law for Civ $\lambda 1549$. In this case (shown in Fig. 8 by small open circles) the correction for Pop. A still implies non-negligible systematic residuals $\delta \log M=\log M_{\mathrm{BH}}\left(\mathrm{H} \beta_{\mathrm{BC}}\right)-$ $\log M_{\mathrm{BH}}(\mathrm{CIV} \lambda 1549) \approx 0.23$. The average residual is higher for Pop. B $M_{\mathrm{BH}}$, with $\delta \log M \approx 0.27$, and scatter $\approx 0.39$ dex.

Assef et al. (2011) used a sample of approximately ten sources with optical spectra covering CIV $\lambda 1549$ and NIR spectra covering $\mathrm{H} \beta$ or $\mathrm{H} \alpha$ and showed that $M_{\mathrm{BH}}$ estimates can be made consistently. The approach of Assef et al. (2011) may also be understood as a correction related to the MS. Assef et al. (2011) suggest that much of the dispersion in their virial mass is caused by the poor correlation between $\lambda L_{\lambda}$ at $5100 \AA$ and at $1350 \AA$ rather than between their line widths. Their Figs. 14 and 15 show that the FWHM Civ $\lambda 1549$ to $\mathrm{H} \beta$ ratio depends on the flux ratio at $1350 \AA$ and $5100 \AA$, which is an MS correlate (Laor et al. 1997; Shang et al. 2011). The Assef et al. 2011 sample of gravitationally lensed quasars might have lowered the Eddington ratio bias described by Sulentic et al. (2014), leading to a preferential section of Pop. B quasars, and better agreement between $\mathrm{H} \beta$ and $\mathrm{CIv} \lambda 1549$ line width.

\subsection{A virialized component}

\subsubsection{Similar $\mathrm{H} \beta$ and $\operatorname{Civ} \lambda 1549$ luminosity trends}

A systematic increase in line width in the HE sample is expected if the line broadening is predominantly virial: Fig. 5 of Paper I shows that there are no FWHM H$\beta \lesssim 3000 \mathrm{~km} \mathrm{~s}^{-1}$ at $\log L \gtrsim$ $47\left[\mathrm{erg} \mathrm{s}^{-1}\right.$ ]. Figure 9 here shows that a similar increase in FWHM as a function of luminosity is occurring in the FOS $+\mathrm{HE}$ sample for both $\mathrm{H} \beta$ and CIV $\lambda 1549$. The FWHM ratio between Civ $\lambda 1549$ and $\mathrm{H} \beta$ does not instead appear strongly influenced by $L$, leading to the interpretation that the broadening of both lines - even if the Civ $\lambda 1549$ centroid measurements are significantly affected by an outflowing component - may be mostly related to the gravitational effects of the supermassive black hole (as further discussed below). As mentioned, Balmer lines provide a VBE up $z \gtrsim 2$, and the results of Paper I extended this finding to the highest luminosities. For Civ $\lambda 1549$, Fig. 9 and the correlation $F W H M-c\left(\frac{1}{2}\right)$ justify the assumption of a virial broadening component coexisting with a nonvirial one (Wang et al. 2011).

\subsubsection{Orientation effects on $\mathrm{H} \beta$}

A large part of the Civ $\lambda 1549-\mathrm{H} \beta$ scatter is expected to be due to orientation effects. The issue of orientation effects remains open for RQ sources, and orientation effects are most likely strongly affecting the FWHM of $\mathrm{H} \beta$ (Mejía-Restrepo et al. 2018b), even if it remains hard to distinguish them from other physical factors (such as $M_{\mathrm{BH}}$ and $L / L_{\mathrm{Edd}}$ ). A clue is given by the $4 \mathrm{DE} 1$ predictions at extreme orientations: objects observed near the disk rotation axis (i.e., nearly pole-on) have the smallest FWHM $\mathrm{H} \beta$, the strongest FerI and Can intensities (Dultzin-Hacyan et al. 1999), the largest soft $X$ excess, and the largest Civ $\lambda 1549$ blue shifts/asymmetries. These predictions are motivated by the physical scenarios involving an accretion disk-wind system. From a pole-on orientation we should see the smallest Doppler broadening of virially dominated $\mathrm{H} \beta$-emitting clouds, and the strongest intensity of FeII and other LILs if they are emitted from clouds in the outer part of the disk (Martínez-Aldama et al. 2015). We should also observe the largest contribution of the soft $\mathrm{X}$ excess if it is related to disk emission (Wang et al. 1996, 2014b; Boller et al. 1996). Finally, if a wind is associated with an optically 

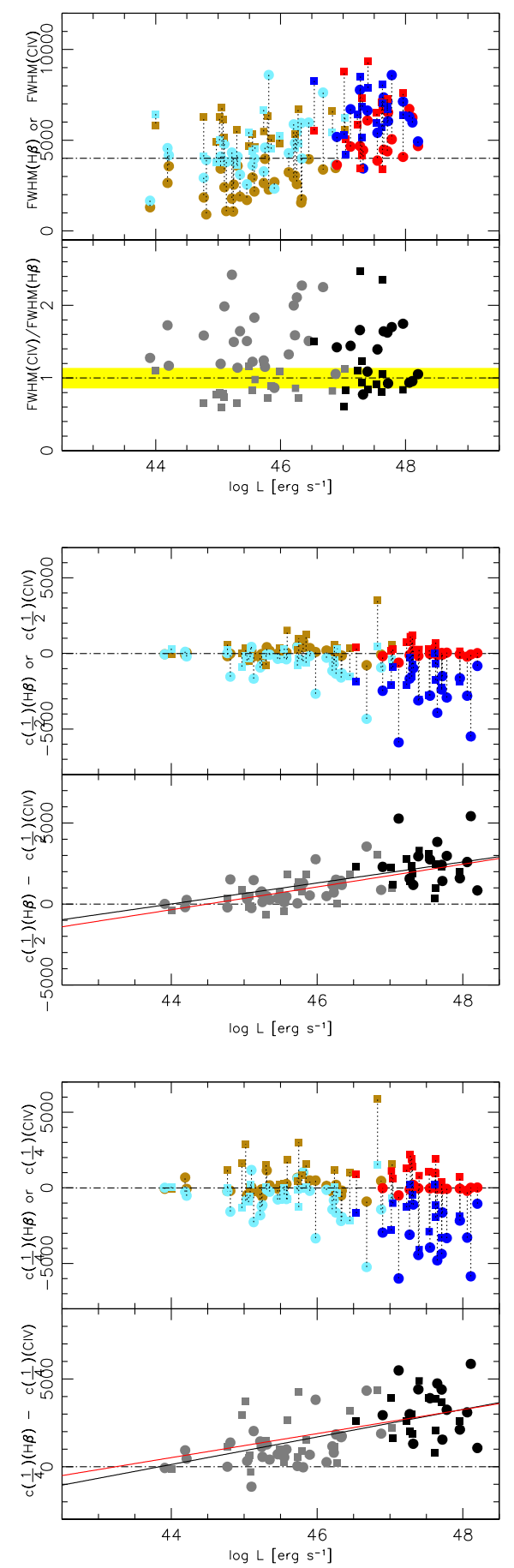

Fig. 9. $\mathrm{H} \beta$ and Civ $\lambda 1549$ profile parameter comparison as a function of luminosity. Top panels: behavior of FWHM Civ $\lambda 1549$ and $\mathrm{H} \beta$ (upper half) and of the ratio $\mathrm{FWHM}(\mathrm{Civ} \lambda 1549) / \mathrm{FWHM}(\mathrm{H} \beta)$ as a function of $L$ (lower half), for FOS (golden and pale blue) and HE sample (red and blue). The yellow band identifies the region where FWHM(CIV 11549$) / F W H M(H \beta)=1$ within the errors. Middle panels: $c\left(\frac{1}{2}\right)$ of $\mathrm{H} \beta$ and Civ $\lambda 1549$ (upper half), and difference $\delta\left(\frac{1}{2}\right)$ as a function of $L$ (lower half). Square symbols indicate Pop. B, circles Pop. A. Lines trace an unweighted least-squares fit for the Pop. A (black) and for Pop. B (red) sources. Bottom panels: as in top panels but for $c\left(\frac{1}{4}\right)$ and $\delta\left(\frac{1}{4}\right)$. The vertical dotted lines join $\mathrm{H} \beta$ and Civ $\lambda 1549$ parameters for the same object (e.g., they are not error bars).

thick disk, and its dynamics is dominated by radiation pressure, HILs such as Civ 11549 emitted in the wind would show the largest blueshifts (if the receding part of the flow is shielded from view). The case of I $\mathrm{Zw} 1$ provided a prototypical case in which a flattened LIL-emitting systems and a radial outflow could be seen at small inclination (e.g., Marziani et al. 1996; Leighly 2004).

From a more modern perspective, there are several indications that the low-ionization BLR is a flattened system (Mejía-Restrepo et al. 2017, 2018a; Negrete et al. 2017, 2018). We see the clearest evidence at the MS extrema: extreme Pop. B sources radiating at very low $L / L_{\text {Edd }}$ frequently show LIL profiles consistent with geometrically thin accretion disk profiles (e.g., Chen \& Halpern 1989; Strateva et al. 2003; Storchi-Bergmann et al. 2017), which may be hidden in the majority of Pop. B sources (Bon et al. 2007, 2009). A highly flattened LIL-BLR is also suggested in blazars, which are also Pop. B low-radiatiors (Decarli et al. 2011), by comparing the virial product to mass estimates obtained from the correlation between $M_{\mathrm{BH}}$ and the host galaxy luminosity. At the other end of the MS, extreme Pop. A quasars show deviations from virial luminosity estimates consistent with the effect of orientation on the line width, if the emitting region is highly flattened (Negrete et al. 2018). A flattened low-ionization BLR is also suggested by comparing the virial product to mass estimates obtained from accretion disk fits to the SED (Mejía-Restrepo et al. 2017, 2018a).

The effect of orientation on the FWHM and on $M_{\mathrm{BH}}$ and $L / L_{\text {Edd }}$ estimates can be computed by assuming that we are observing randomly oriented samples of quasars whose line emission arises from a flattened structure - possibly the accretion disk itself. The probability of viewing the structure with an isotropic velocity broadening $\delta v_{\text {iso }}$ at an angle $\theta$ between line-of-sight and the symmetry axis of a flattened structure is $P(\theta)=\sin (\theta)$. The radial velocity spread (in the following we use the FWHM as a measure, $\delta v_{\mathrm{obs}}=F W H M$ ) can be written as

$$
\frac{F W H M^{2}}{4}=\delta v_{\text {iso }}^{2}+\delta v_{\mathrm{K}}^{2} \sin ^{2} \theta
$$

which implies that

$\frac{M_{\mathrm{BH}, \mathrm{obs}}}{M_{\mathrm{BH}, \mathrm{K}}}=\frac{\delta v_{\mathrm{obs}}^{2}}{\delta v_{\mathrm{K}}^{2}}=4 \cdot\left(\kappa^{2}+\sin ^{2} \theta\right)$,

where $\kappa=\delta v_{\text {iso }} / \delta v_{\mathrm{K}}$. From Eq. (9) one can estimate the ratio of $\delta v_{\mathrm{obs}}$ to intrinsic velocity $\delta v_{\mathrm{K}}$ either by computing the most probable value of $\theta$ or by deconvolving the observed velocity distribution from $P(\theta)$. The calculations are described in Appendix B. The average ratio is $\left\langle\frac{M_{\mathrm{BH}, \mathrm{obs}}}{M_{\mathrm{BH}, \mathrm{K}}}\right\rangle \approx 1.1$ if $\kappa=0.1$. If the FWHM of the $\mathrm{H} \beta$ line is used, the $M_{\mathrm{BH}}$ suffers from a small bias if the LIL-emitting region is highly flattened. If $\kappa=0.5$ (a "fat" emitting region), then the bias is much larger $\left\langle\frac{M_{\mathrm{BH}, \mathrm{obs}}}{M_{\mathrm{BH}, \mathrm{K}}}\right\rangle \approx 2.1$. The $M_{\mathrm{BH}}$ dispersion in the case of $\kappa=0.1$ was estimated for large samples $\left(10^{6}\right.$ replications) with $\theta$ distributed according to $P(\theta)$ (Appendix C), and was found to be $\sigma_{M_{\mathrm{BH}}} \approx 0.33$ dex. Therefore, even if $P(\theta)$ strongly disfavors cases with $\theta \rightarrow 0$, the viewing angle can account for a large fraction of the dispersion in the $M_{\mathrm{BH}}$ scaling laws with line width and luminosity.

\subsection{A wind component}

The interpretation of the CIV $\lambda 1549$ profile (and $\mathrm{H} \beta$ profile differences) rests on the main results of Paper I: the Civ $\lambda 1549$ shifts are dependent on $L / L_{\text {Edd }}$ and, to a lesser extent on $L$; the CIv $\lambda 1549$ broadening is due to a blueshifted component whose strength with respect to a virialized component increases with $L / L_{\text {Edd }}$ and $L$. 
At one quarter and one half of the fractional intensity the difference in the line centroid radial velocity of $\mathrm{H} \beta$ and Civ $\lambda 1549$, that is, $c\left(\frac{1}{2}\right)(\mathrm{H} \beta)-c\left(\frac{1}{2}\right)(\mathrm{CIV} \lambda 1549)$ and $c\left(\frac{1}{4}\right)(\mathrm{H} \beta)-$ $c\left(\frac{1}{4}\right)$ (Civ 11549$)$, is almost always positive, and can reach $7000 \mathrm{~km} \mathrm{~s}^{-1}$ and $4000 \mathrm{~km} \mathrm{~s}^{-1}$ in the HE sample and FOS, respectively, mainly because of the large Civ $\lambda 1549$ blueshifts (Fig. 9). A luminosity dependence of $\delta\left(\frac{1}{2}\right)=c\left(\frac{1}{2}\right)(\mathrm{H} \beta)-$ $c\left(\frac{1}{2}\right)(\operatorname{CIv} \lambda 1549)$ and $\delta\left(\frac{1}{4}\right)=c\left(\frac{1}{4}\right)(\mathrm{H} \beta)-c\left(\frac{1}{4}\right)(\operatorname{CIV} \lambda 1549)$ is illustrated in Fig. 9. The centroid separations are correlated with $L$, with a similar slope at both one quarter and one half of the fractional intensity (Fig. 9): for $\delta\left(\frac{1}{2}\right)$ of Pop. A,

$\delta\left(\frac{1}{2}\right) \approx(648 \pm 121) \log L-(28530 \pm 5600) \mathrm{kms}^{-1}$,

in the range $44 \lesssim \log L \lesssim 48.5$.

The trends of Fig. 9 suggest that the Civ $\lambda 1549$ broadening is however affected by $M_{\mathrm{BH}}$, as both the $\mathrm{H} \beta$ and Civ $\lambda 1549$ widths steadily increase with luminosity, and their ratio shows no strong dependence on luminosity. This may be the case if the outflow velocity is a factor $k$ of the virial velocity $(k=\sqrt{2}$ would correspond to the escape velocity). The correlation between shift and FWHM of Paper I indicates that we are seeing an outflow component "emerging" on the blue side of the BC. If we assume that line emission arises from a flattened structure with velocity dispersion $v_{\text {iso }}$ (i.e., as in Eq. (9)), and that the outflowing component from the accretion disk contributes to an additional broadening term proportional to $\cos \theta$ (the projection along the line of sight of the outflow velocity), then the observed Civ $\lambda 1549$ broadening can be written as

$F W H M_{\mathrm{CIV}}^{2}=4\left(\delta v_{\text {iso }}^{2}+\delta v_{\mathrm{K}}^{2} \sin ^{2} \theta\right)+\jmath^{2} \delta v_{\mathrm{K}}^{2} M \frac{L}{L_{\mathrm{Edd}}} \cos ^{2} \theta$,

where $\mathrm{I}$ is a proportionality constant, and $\mathcal{M}$ the force multiplier. It follows that the total broadening can easily exceed $\delta v_{\mathrm{K}}$ for a typical viewing angle $\theta=\pi / 6$, provided that the factor $Q=\beth^{2} \mathcal{M} \frac{L}{L_{\mathrm{Edd}}}$ is larger than 1 . The factors $\$ and $\mathcal{M}$ depend on physical properties (density, ionization level) and should be calculated in a real physical model linking ionization condition and dynamics. The factor $Q$ encloses the dependence of wind properties on radiation forces, opacity, and so on (Stevens \& Kallman 1990) along with the dependence on ionization. For example, in the case of optically thick gas being accelerated by the full absorption of the ionizing continuum, the force multiplier is $\mathcal{M}=\frac{\alpha}{\sigma_{\mathrm{T}} N_{\mathrm{c}}} \approx 7.5$ for column density $N_{\mathrm{c}}=10^{23} \mathrm{~cm}^{-2}$, and $\alpha=0.5(\alpha$ is the fraction between the ionizing and bolometric luminosity, Netzer \& Marziani 2010). If $L / L_{\mathrm{Edd}} \rightarrow 1$, and $\ \sim 1$, implying $Q \sim O(10)$, the FWHM ${ }_{\text {CIV }}$ can exceed the $\operatorname{FWHM}(\mathrm{H} \beta)$ by up to a factor of several, as indeed observed in the most extreme radiators from the comparison between Civ $\lambda 1549$ and $\mathrm{H} \beta$.

Equations (9) and (12) account for the consistent increase in broadening of Civ $\lambda 1549$ and $\mathrm{H} \beta$ (Fig. 9). In the context of the present sample covering a wide range in luminosity, $L$ can be considered a proxy for the increase in $M_{\mathrm{BH}}\left(L \propto M_{\mathrm{BH}}\right.$, with a scatter set by the $L / L_{\mathrm{Edd}}$ distribution) and therefore in Keplerian velocity. The top and middle panels of Fig. 9 show a consistent increase of the centroid, and of the centroid difference $\delta\left(\frac{1}{2}\right)$ and $\delta\left(\frac{1}{4}\right)$ with $L$. This result motivated the introduction of a luminosity-dependent correction to the line width. The centroid difference can be written as

$\delta\left(\frac{i}{4}\right) \sim \frac{1}{2} \delta v_{\mathrm{K}}\left(\frac{i}{4}\right) \cos \theta\left(-\mathrm{J}\left(M \frac{L}{L_{\mathrm{Edd}}}\right)^{\frac{1}{2}}+f\right), i=1,2$, with $f \equiv 0$ for Pop. A, and $f$ defined by the infall velocity $v_{\text {inf }}=f \delta v_{\mathrm{K}}$ as a fraction of the radial free-fall velocity for Pop. B. Equations (13) and (12) imply that $F W H M_{\mathrm{CIV}}^{2}=$ $F W H M_{\mathrm{H} \beta}^{2}+4 \delta^{2}\left(\frac{1}{2}\right)$, if $f=0$.

If we ascribe the redward displacement of the $\mathrm{H} \beta$ wing in Pop. B sources to gravitational and transverse redshift (e.g., Corbin 1990; Bon et al. 2015),

$$
\begin{aligned}
\delta\left(\frac{i}{4}\right) & =\frac{1}{2}\left(-\delta v_{\mathrm{K}}\left(\frac{i}{4}\right) \mathrm{J}\left(\mathcal{M} \frac{L}{L_{\mathrm{Edd}}}\right)^{\frac{1}{2}} \cos \theta+\frac{3}{2} c z_{\mathrm{g}}\left(\frac{i}{4}\right)\right) \\
& =\frac{1}{2} \delta v_{\mathrm{K}}\left(\frac{i}{4}\right)\left(-\mathrm{J}\left(\mathcal{M} \frac{L}{L_{\mathrm{Edd}}}\right)^{\frac{1}{2}} \cos \theta+\frac{3}{2} \frac{\delta v_{\mathrm{K}}\left(\frac{i}{4}\right)}{c}\right),
\end{aligned}
$$

where $c z_{\mathrm{g}} \sim c G M_{\mathrm{BH}} / c^{2} r$ is the $c\left(\frac{1}{2}\right)$ or $c\left(\frac{1}{4}\right)$ of $\mathrm{H} \beta$, which can usually be 0 (Pop. A) or $\geq 0$ (Pop. B), and where we have used the weak field approximation for the gravitational redshift.

Equations (13) and (14) account for the steady increase in the centroid difference $\delta$ with luminosity. The $\mathrm{H} \beta$ centroid displacement in Pop. B may be associated with free fall or gravitational redshift. The amplitude of blueshift depends on luminosity in Pop. A. The point is that both $\mathrm{H} \beta$ redward displacement and blueshift of CIV $\lambda 1549$ (and hence their differences) are proportional to the $\delta v_{\mathrm{K}}$ and hence to the $M_{\mathrm{BH}}$.

The $\xi_{\mathrm{CIV}}$ factor can be written as

$$
\begin{aligned}
\xi_{\mathrm{CIV}} & =\left(\frac{\delta v_{\text {iso }}^{2}+\delta v_{\mathrm{K}}^{2} \sin ^{2} \theta}{4\left(\delta v_{\text {iso }}^{2}+\delta v_{\mathrm{K}}^{2} \sin ^{2} \theta\right)+\jmath^{2} \delta v_{\mathrm{K}}^{2} \mathcal{M} \frac{\mathcal{L}}{\mathcal{L}_{\mathrm{Edd}}} \cos ^{\epsilon} \theta}\right)^{\frac{1}{2}} \\
& =\left(\frac{1}{1+\frac{Q \cos ^{2} \theta}{4\left(\kappa^{2}+\sin ^{2} \theta\right)}}\right)^{\frac{1}{2}} .
\end{aligned}
$$

The $\xi_{\text {CIV }}$ behavior as a function of the viewing angle $\theta$ is described in the top panel of Fig. 10. The figure shows the dependence in the case of a flat $\kappa=0.1$ (black) or fat $\kappa=0.5$ (red) for four values of $Q$. The bottom panel of Fig. 10 shows the behavior of the ratios $\tilde{\xi}_{\mathrm{H}} \beta=1 /\left[4\left(\kappa^{2}+\sin ^{2} \theta\right)\right]^{1 / 2}$ and $\tilde{\xi}_{\mathrm{CIV}}=$ $1 /\left(4 \kappa^{2}+4 \sin ^{2} \theta+Q \cos ^{2} \theta\right)^{1 / 2}$. The $\tilde{\xi}$ are the ratios between the $\delta v_{\mathrm{K}}$ and the observed FWHM. At low $\theta$, the FWHM(H $\left.\beta\right)$ underestimates the $\delta v_{\mathrm{K}}$ by a large factor, while the overestimation of $\delta v_{\mathrm{K}}$ by the FWHM(CIV $\left.\lambda 1549\right)$ is almost independent of $\theta$ and a factor $\approx 2$.

The panels of Fig. 11 compare the observed distribution of $\xi_{\mathrm{CIV}, 1}$ (shaded histogram) with the prediction of randomly oriented synthetic samples, at different $Q$. We are not seeking a fit of the observed distribution especially around $\xi_{\mathrm{CIV}} \approx 1$ because of the many biases affecting our sample and of the problem raised by $\xi_{\mathrm{CIV}}>1$ (see below), but a qualitative consistency in the distribution of $\xi_{\mathrm{CIV}}<1$.

If we focus the analysis of Fig. 11 mainly on large shifts, the presence of low $\xi_{\text {CIV }}$ values and their higher frequency favors a highly flattened low-ionization BLR, as well as high $Q$ for the full sample. A fat $\kappa=0.5 \mathrm{BLR}$ is unable to reproduce the largest shift amplitudes. The scatter in $\xi_{\mathrm{CIV}}$ linear values at $Q \gtrsim 2$ is $\approx 0.2$, implying a dispersion in the $M_{\mathrm{BH}}$ of $\approx 0.15$ dex. If we separate Pop. $\mathrm{A}$ and $\mathrm{B}$, more extreme values of $Q \gtrsim 2$ are required to fit the large shift distribution in Pop. A, with $Q \sim 10$. The distribution of $\xi_{\mathrm{CIV}}$ for Pop. B is more peaked around $\xi_{\mathrm{CIV}} \approx 1$, and the $\xi_{\text {CIV }}$ distribution can be qualitatively accounted for if $Q \lesssim 2$.

The $\xi_{\text {CIV }}$ observed distribution includes values $>1$. These values are not possible following our model: the FWHM(Civ 11549$)$ should be always in excess of or comparable 

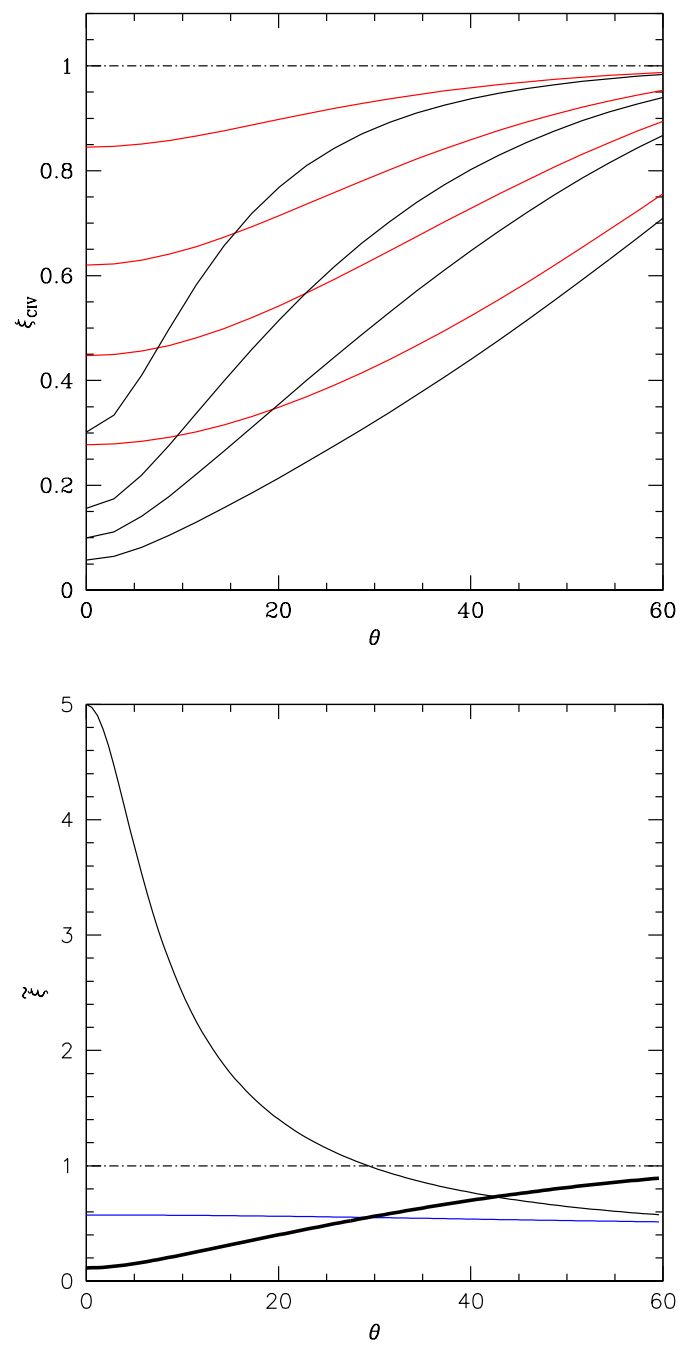

Fig. 10. Top panel: parameter $\xi_{\text {CIV }}$ behavior as a function of viewing angle as a function of $\theta$ for a "thin" emitting region with $\kappa=0.1$, for different $Q$ values $(0.4,1.6,4.0,12.0$; black lines $)$. Red line: $\xi_{\text {CIV }}$ behavior for a thick emitting region $\kappa=0.5$, for the same $Q$ values. Bottom panel: as in top panel but for $K=0.1$, with $Q=12$. The thin lines are the $\tilde{\xi}$ values for $\mathrm{H} \beta$ (black) and Civ $\lambda 1549$ (blue). See text for more details. The thick line is their ratio (also shown in the top panel).

to $\operatorname{FWHM}\left(\mathrm{H} \beta_{\mathrm{BC}}\right)$. In the case of $Q \gg 1$, the CIV $\lambda 1549$ line is broadened by an outflowing component; if $Q \rightarrow 0, \xi_{\text {CIV }} \lesssim 1$. In the latter case, the excess broadening may come from the smaller emissivity-weighted distance expected for Civ $\lambda 1549$ in a virial velocity field. The existence of cases with FWHM Civ $\lambda 1549<$ FWHM H $\beta$ was already noted by Mejía-Restrepo et al. (2018b), and therefore it is not unique to the FOS+HE sample. The bottom-right panel of Fig. 11 shows that such cases are relatively frequent among Pop. B. Inspection of the HE spectra in Paper I reveals that the Civ $\lambda 1549$ profile is significantly affected by semi-broad absorptions such as the ones often found in mini-BAL quasars (Vestergaard 2003; Sulentic et al. 2006b). Since mini-BALs cluster around the line core, it is most likely that these Pop. B sources would satisfy the condition FWHM CIV $\lambda 1549 \gtrsim \mathrm{FWHM} \mathrm{H} \beta$ if the effect of the absorptions could be removed.

\section{4. $M_{B H}$ scaling laws dependent on $L / L_{E d d}$ and $L$}

The goal is to obtain an $M_{\mathrm{BH}}$ estimator based on Civ $\lambda 1549$ that is consistent with the scaling law derived for $\mathrm{H} \beta$. In this context,
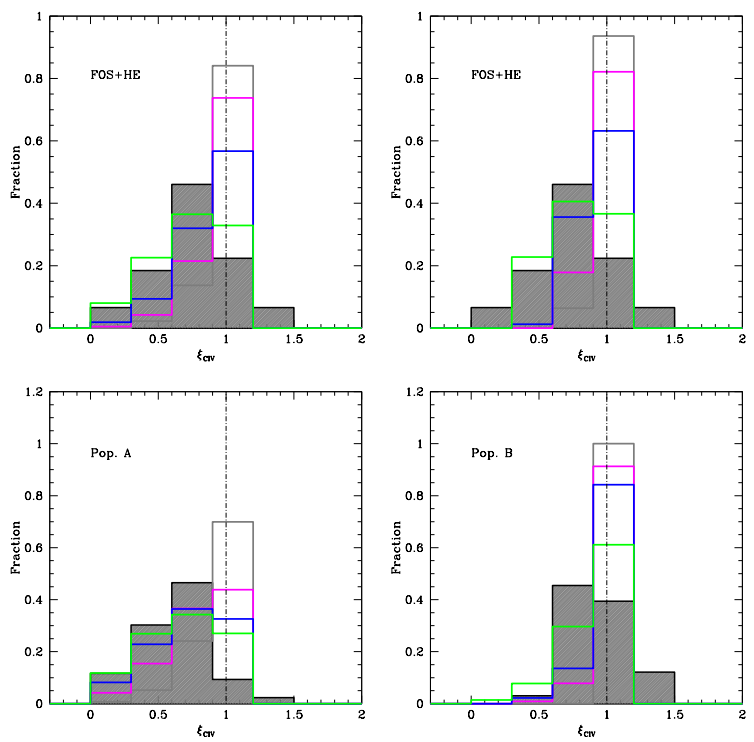

Fig. 11. Top left: observed distribution of $\xi_{\mathrm{CIV}}$ for the full FOS+HE sample (shaded histogram), and distribution of $\xi_{\mathrm{CIV}}$ for $Q=0.4$ (gray), 0.8 (magenta), 2.0 (blue), and 7.8 (green), assuming $\kappa=0.1$, for randomly oriented synthetic samples. Top right: as in top-left panel but for $\kappa=0.5$. Bottom left: distribution of $\xi_{\text {CIV }}$ restricted to Pop. A sources for $Q=1.0$ (gray), 2.0 (magenta), 4.0 (blue), and 12.0 (green), for $\kappa=0.1$. Bottom right: as in bottom-left but for Pop. B sources, with $Q=0$. (gray), 0.2 (magenta), 0.4 (blue), and 1.6 (green), for $\kappa=0.1$. See text for more details.

the dependence on luminosity of FWHM Civ $\lambda 1549$ cannot be ignored especially if samples encompass a broad range in luminosity. This will be the case in deep, forthcoming surveys. Considering the corrections to FWHM CIV $\lambda 1549$ of Sect. 4, the $M_{\mathrm{BH}}$ scaling law is derived in the form $\log M_{\mathrm{BH}}=\alpha \cdot \log L+2$. $\log F W H M+\gamma$ by minimizing the scatter and any systematic deviation of $M_{\mathrm{BH}}$ estimated from Civ $\lambda 1549$ with respect to the $\mathrm{H} \beta$-derived masses: the unweighted least-square fit of Fig. 12 yields

$M_{\mathrm{BH}}(\mathrm{CIV}) \approx(0.99 \pm 0.04) M_{\mathrm{BH}}(\mathrm{H} \beta)+(0.11 \pm 0.39)$.

The Civ $\lambda 1549$ scaling law, with $M_{\mathrm{BH}}$ in solar units and $L_{1450}$ normalized by $10^{44}$, takes the form:

$$
\begin{aligned}
\log M_{\mathrm{BH}, 1} \mathrm{CIV} \approx & \left(0.64_{-0.025}^{+0.045}\right) \log L_{1450,44} \\
& +2 \log \left(\xi_{\mathrm{CIV}, 1} F W H M(\mathrm{CIV})\right)+\left(0.525_{-0.18}^{+0.22}\right)
\end{aligned}
$$

for the FWHM correction using Eq. (6). Applying Eq. (7), the scaling law does not change appreciably, and uncertainties in the coefficients are only slightly different.

$$
\begin{aligned}
\log M_{\mathrm{BH}, 2} \mathrm{CIV} \approx & \left(0.63_{-0.035}^{+0.045}\right) \log L_{1450,44} \\
& +2 \log \left(\xi_{\mathrm{CIV}, 2} \mathrm{FWHM}(\mathrm{CIV})\right)+\left(0.525_{-0.19}^{+0.275}\right)
\end{aligned}
$$

The scaling law parameter uncertainties have been estimated following the standard approach in Bevington \& Robinson (2003, p. 210ff), with the constraint that unbiased consistency between $M_{\mathrm{BH}}$ from $\mathrm{H} \beta$ and $\mathrm{Crv} \lambda 1549$ (Eq. (16)) is satisfied within the $1 \sigma$ uncertainties. The rms scatter is $\sigma \approx 0.33$ for Eq. (6) and $\sigma \approx 0.35$ for Eq. (7). Assuming a single correction for both Pop. A and B significantly worsens the fit quality, and no scaling law is reported. 

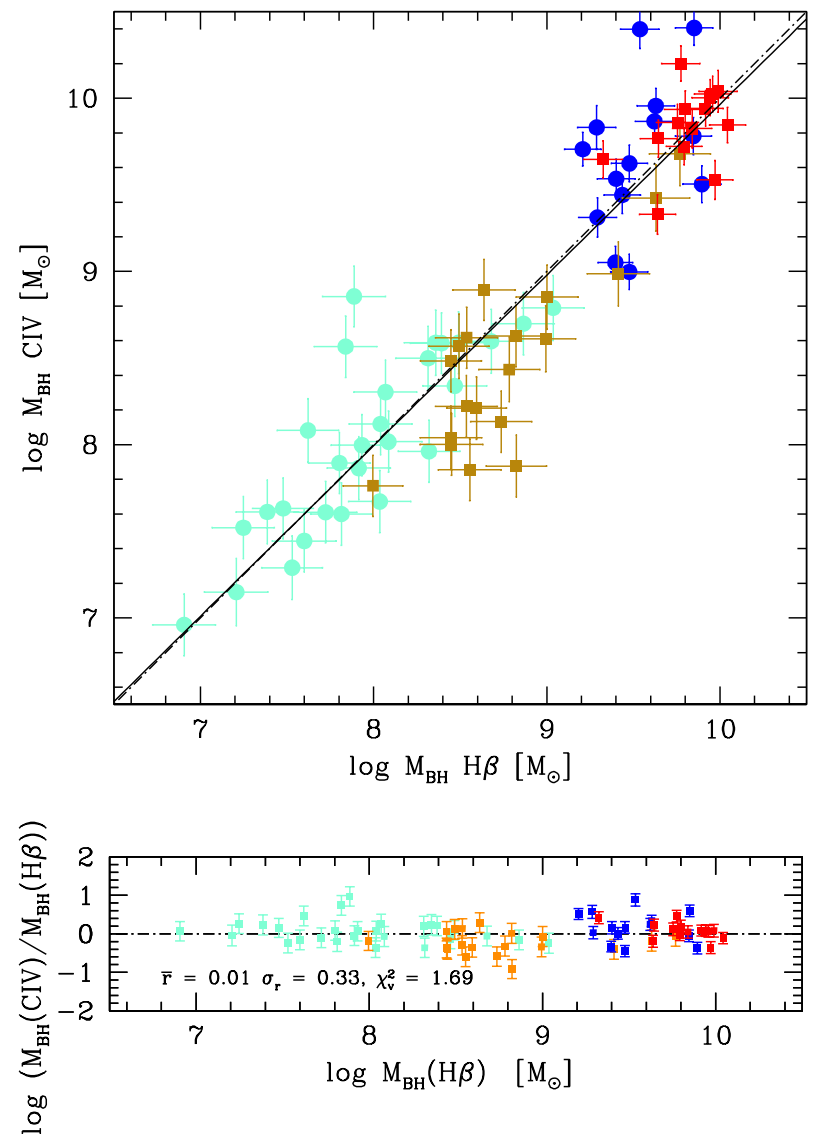

Fig. 12. $M_{\mathrm{BH}}$ computed from the fiducial relation of Vestergaard \& Peterson (2006) based on FWHM H $\beta$ vs. the one computed from the Civ $\lambda 1549$ FWHM corrected following Eq. (6). Error bars include luminosity uncertainties estimated by the scatter in $L$ derived from the UV and the visual spectral ranges along with errors on FWHM propagated quadratically. Lower panel: residuals for the three cases. Meaning of the color-code is the same as in the previous Figs.

An application of the bisector-fitting technique using SLOPES (Feigelson \& Babu 1992) yields

$$
\begin{aligned}
\log M_{\mathrm{BH}, 1} \mathrm{CIV} \approx & \left(0.5925_{-0.030}^{+0.0275}\right) \log L_{1450,44} \\
& +2 \log \left(\xi_{\mathrm{CIV}, 1} F W H M(\mathrm{CIV})\right)+(0.62 \pm 032)
\end{aligned}
$$

$\log M_{\mathrm{BH}, 2} \mathrm{CIV} \approx\left(0.572_{-0.032}^{+0.0285}\right) \log L_{1450,44}$

$$
+2 \log \left(\xi_{\mathrm{CIV}, 2} F W H M(\mathrm{CIV})\right)+(0.64 \pm 035) \text {. }
$$

The Vestergaard \& Peterson (2006) $\mathrm{H} \beta$ scaling laws suffer from significant scatter (see the discussion in their paper) that can be explained on the basis of the scatter induced by orientation $(0.33$ dex at $1 \sigma)$ according to the results of Appendix B. The Civ $\lambda 1549$ and $\mathrm{H} \beta$ relation should be considered equivalent. The luminosity exponent $(\approx 0.64)$ is in agreement with previous observations (Peterson et al. 2005). It is slightly above the exponent of the Civ $\lambda 1549$ radius dependence on luminosity found in more recent reverberation mapping studies $(\approx 0.52-0.55$ Kaspi et al. 2007; Lira et al. 2017, 2018).

Figure 12 suggests the presence of a well-behaved distribution with a few outlying points. It is possible to reduce the scatter to $\sigma \approx 0.25$ applying a $\sigma$ clipping algorithm (i.e., eliminating all sources deviating more than $\pm 2 \sigma$ ), with no significant change in the best-fit parameters. This selective procedure is

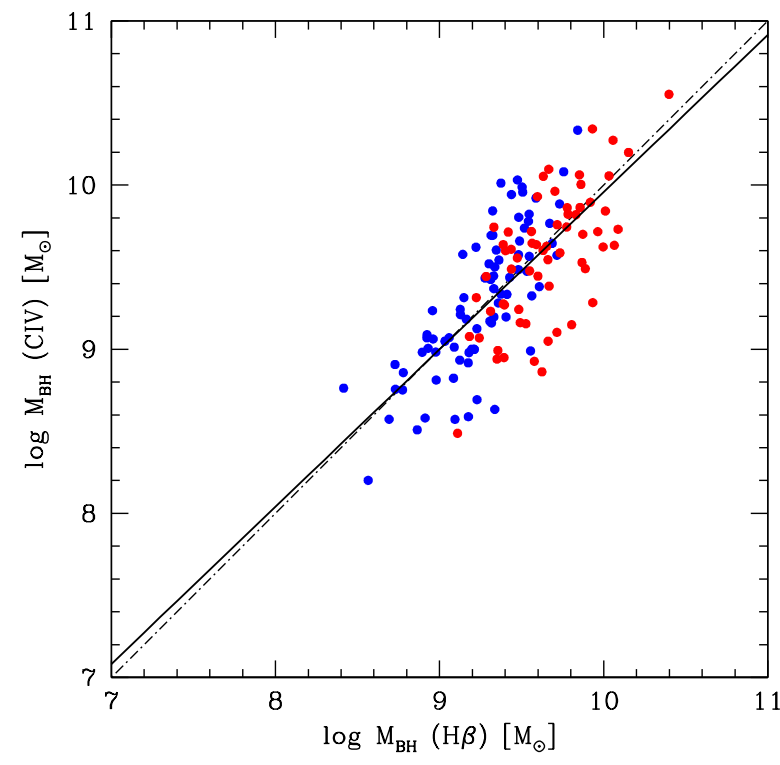

Fig. 13. Estimates of $M_{\mathrm{BH}}$ using corrected Civ $\lambda 1549$ FWHM as a VBE versus $M_{\mathrm{BH}}$ computed from $\mathrm{H} \beta$ FWHM using the scaling law of Vestergaard \& Peterson (2006). The luminosity and FWHM(Civ $\lambda 1549)$ data from Coatman et al. (2017) for Pop. A (blue) and B sources (red; see Sect. 5.5 for more details) are used to compute the Civ $\lambda 1549$ $M_{\mathrm{BH}}$ according to Eq. (17). The dot-dashed line traces the 1:1 relation between $M_{\mathrm{BH}}$ from CIv $\lambda 1549$ and $\mathrm{H} \beta$; the black line is an unweighted least-squares fit for the $\mathrm{A}+\mathrm{B} M_{\mathrm{BH}}$ estimates.

however unwarranted: as shown in Appendix C, outlying points are expected precisely because of the possible occurrence of low-probability viewing angles. The residual rms can be largely accounted for by orientation effects if $Q$ is small, and by the combination of orientation effects and outflow prominence if $Q$ is much larger than 1 (Appendix C).

\subsection{Application to a large sample with $H \beta$ and CIv 1549 data}

The Coatman et al. (2017) data provide a different sample for the testing of the scaling law of Eq. (17). The flux bisector can be converted into $c\left(\frac{1}{4}\right)$ (Sect. 4.5). An application of Eq. (17) to Pop. A and B (applying the luminosity-dependent separation as in Paper I) yields agreement with the expectation of an unbiased $M_{\mathrm{BH}}$ estimator with respect to the $\mathrm{H} \beta M_{\mathrm{BH}}$ estimates using the scaling law of Vestergaard \& Peterson (2006): the slope of an unweighted least-squares fit is $\approx 0.958 \pm 0.065$ (using SLOPES, Feigelson \& Babu 1992, Fig. 13).

\section{Conclusion}

The present investigation focuses on the Civ $\lambda 1549$ relations to $\mathrm{H} \beta$ over a broad range of luminosities ( $\log L \sim 43-48$, including very high luminosities, $\log L \gtrsim 47$ ) in the Eigenvector 1 context, with the goal of testing the CIv $\lambda 1549$ suitability as virial broadening estimators when observations of low-ionization lines are not available. The Eigenvector 1 context means that the quasar main sequence is considered to properly interpret firstorder Eddington ratio effects and luminosity effects that appear to be second order in low- redshift samples.

The main conclusions that we draw from this study are as follows.

1. Within the limits of our sample size, and of our UV spectral coverage, it does not appear that the Civ 21549 FWHM can 
be used as a reliable virial broadening estimator without significant corrections. There is large scatter between FWHM measurements on $\mathrm{H} \beta$ and $\mathrm{CIV} \lambda 1549$ that seems to defy the definition of a meaningful trend.

2. The Civ $\lambda 1549_{\mathrm{NC}}$ removal improves the agreement between CIv $\lambda 1549$ and $\mathrm{H} \beta$ measurements in the HE sample, but Civ $\lambda 1549_{\mathrm{NC}}$ is not a major factor hampering the definition of a Civ $\lambda 1549$ VBE consistent with $\mathrm{H} \beta$.

3. Corrections to FWHM CIV $\lambda 1549$ - and Civ $\lambda 1549-$ based $M_{\mathrm{BH}}$ estimates that vary systematically along the 4DE1 sequence and are strongly dependent on Eddington ratio are promising and should be further explored.

4. Following the results of Paper I, we define a correction to the FWHM(Civ $\lambda 1549)$ based on the full-profile Civ $\lambda 1549 c\left(\frac{1}{2}\right)$ (a proxy for $L / L_{\text {Edd }}$ ) and on the luminosity at $1450 \AA$. Given the intrinsic differences between Pop. A and B, and their "threshold" separation dependent on a critical $L / L_{\text {Edd }}$, two different correction laws were considered for the two populations. We note that the correction for Pop. B as derived from the FOS $+\mathrm{HE}$ sample is highly uncertain.

5. The $M_{\mathrm{BH}}$ scaling law (Eq. (17)) associated with the corrected FWHM(CIV 11549$)$ following Eq. (6), as explained in Sect. 5.4, allows for the preservation of the virial dependence on line broadening. Its practical usefulness rests on the ability to distinguish Pop. A and B quasars. This can be achieved in a large fraction of quasars following the guideline set forth by Negrete et al. (2014).

6 . We constructed a toy model that helped the interpretation of the scatter. Orientation effects induce scatter $\approx 0.3-0.4$ dex in mass estimates that account for a large fraction of the dispersion in the landmark scaling law of Vestergaard \& Peterson (2006). A physical model of the disk + wind system might allow for the viewing angle $\theta$ to be recovered for individual quasars.

Acknowledgements. The authors acknowledge the contribution of Jack Sulentic to the development of this paper which is based on observations made with ESO Telescopes at the Paranal Observatory under programme 082.B-0572(A), and with the Italian Telescopio Nazionale Galileo (TNG) operated on the island of La Palma by the Fundación Galileo Galilei of the INAF at the Spanish Observatorio del Roque de los Muchachos of the Instituto de Astrofísica de Canarias.. PM acknowledges the Programa de Estancias de Investigación (PREI) No. DGAP/DFA/2192/2018 of UNAM, where this paper was completed. The relevant research is part of the projects 176001 "Astrophysical spectroscopy of extragalactic objects" and 176003 "Gravitation and structure of universe on large scales" supported by the Ministry of Education, Science and Technological Development of the Republic of Serbia. MLMA acknowledges a CONA CyT postdoctoral fellowship, and wishes to thank the IAA for support during her postdoctoral stay from February 2016 to July 2018. MLMA acknowledges a CONACyT postdoctoral fellowship and National Science Centre, Poland, grant No.2017/26/A/ST9/00756 (Maestro 9). AdO and MLMA acknowledge financial support from the Spanish Ministry of Economy and Competitiveness through grant AYA2016-76682-C3-1-P. AdO acknowledges financial support from the State Agency for Research of the Spanish MCIU through the "Center of Excellence Severo Ochoa" award for the Instituto de Astrofísica de Andalucía (SEV-2017-0709). MLMA, PM and MDO acknowledge funding from the INAF PRIN-SKA 2017 program 1.05.01.88.04. DD and AN acknowledge support from CONACyT through grant CB221398. DD also thanks for support from grants IN108715 and IN113719 PAPIIT, DGAPA, UNAM. MAMC was partially supported by the Spanish Research project MTM2015-64095-P and by Diputación General de Aragón, Group E24-17R. The scientific results reported in this article are also based on publicly available HST spectra.

\section{References}

Assef, R. J., Denney, K. D., Kochanek, C. S., et al. 2011, ApJ, 742, 93 Bañados, E., Venemans, B. P., Mazzucchelli, C., et al. 2018, Nature, 553, 473

Bachev, R., Marziani, P., Sulentic, J. W., et al. 2004, ApJ, 617, 171

Baldwin, J. A., Ferland, G. J., Korista, K. T., et al. 1996, ApJ, 461, 664
Bensch, K., del Olmo, A., Sulentic, J., Perea, J., \& Marziani, P. 2015, JApA, 36, 467

Bevington, P. R., \& Robinson, D. K. 2003, Data Reduction and Error Analysis for the Physical Sciences (McGraw-Hill)

Bischetti, M., Piconcelli, E., Vietri, G., et al. 2017, A\&A, 598, A122

Bisogni, S., di Serego Alighieri, S., Goldoni, P., et al. 2017, A\&A, 603, A1

Boller, T., Brandt, W. N., \& Fink, H. 1996, A\&A, 305, 53

Bon, E., Popović, L., \& Gavrilović, N. 2007, in Spectral Line Shapes in Astrophysics, eds. L. C. Popovic, \& M. S. Dimitrijevic, AIP Conf. Ser., 938, 59

Bon, E., Popović, L. Č., Gavrilović, N., Mura, G. L., \& Mediavilla, E. 2009, MNRAS, 400, 924

Bon, N., Bon, E., Marziani, P., \& Jovanović, P. 2015, Ap\&SS, 360, 7

Boroson, T. A., \& Green, R. F. 1992, ApJS, 80, 109

Boyle, B. J., Shanks, T., Croom, S. M., et al. 2000, MNRAS, 317, 1014

Brotherton, M. S., Wills, B. J., Steidel, C. C., \& Sargent, W. L. W. 1994a, ApJ, 423, 131

Brotherton, M. S., Wills, B. J., Francis, P. J., \& Steidel, C. C. 1994b, ApJ, 430, 495

Brotherton, M. S., Runnoe, J. C., Shang, Z., \& DiPompeo, M. A. 2015, MNRAS, 451, 1290

Burbidge, G. R., \& Burbidge, E. M. 1967, Quasi-stellar Objects (San Francisco: Freeman)

Chen, K., \& Halpern, J. P. 1989, ApJ, 344, 115

Coatman, L., Hewett, P. C., Banerji, M., \& Richards, G. T. 2016, MNRAS, 461, 647

Coatman, L., Hewett, P. C., Banerji, M., et al. 2017, MNRAS, 465, 2120

Collin-Souffrin, S., Dyson, J. E., McDowell, J. C., \& Perry, J. J. 1988, MNRAS, 232, 539

Corbin, M. R. 1990, ApJ, 357, 346

Corbin, M. R., \& Boroson, T. A. 1996, ApJS, 107, 69

Croom, S. M. 2011, ApJ, 736, 161

Czerny, B., \& Hryniewicz, K. 2011, A\&A, 525, L8

Debuhr, J., Quataert, E., \& Ma, C.-P. 2012, MNRAS, 420, 2221

Decarli, R., Dotti, M., \& Treves, A. 2011, MNRAS, 413, 39

Denney, K. D., Peterson, B. M., Pogge, R. W., et al. 2010, ApJ, 721, 715

Denney, K. D., Vestergaard, M., Watson, D., \& Davis, T. 2012, Am. Astron. Soc.

Meeting Abstracts, 219, 440.20

Denney, K. D., Pogge, R. W., Assef, R. J., et al. 2013, ApJ, 775, 60

Denney, K. D., Horne, K., Brandt, W. N., et al. 2016, ApJ, 833, 33

Du, P., Lu, K.-X., Hu, C., et al. 2016, ApJ, 820, 27

Du, P., Zhang, Z.-X., Wang, K., et al. 2018, ApJ, 856, 6

Dultzin-Hacyan, D., Taniguchi, Y., \& Uranga, L. 1999, in Structure and Kinematics of Quasar Broad Line Regions, eds. C. M. Gaskell, W. N. Brandt, M. Dietrich, D. Dultzin-Hacyan, \& M. Eracleous, ASP Conf. Ser., 175, 303 Elvis, M. 2000, ApJ, 545, 63

Eracleous, M., \& Halpern, J. P. 2004, ApJS, 150, 181

Feigelson, E. D., \& Babu, G. J. 1992, ApJ, 397, 55

Ferland, G. J., Hu, C., Wang, J., et al. 2009, ApJ, 707, L82

Fine, S., Croom, S. M., Bland-Hawthorn, J., et al. 2010, MNRAS, 409, 591

Flohic, H. M. L. G., Eracleous, M., \& Bogdanović, T. 2012, ApJ, 753, 133

Gallerani, S., Fan, X., Maiolino, R., \& Pacucci, F. 2017, PASA, 34, e022

Gaskell, C. M. 1982, ApJ, 263, 79

Goad, M. R., \& Korista, K. T. 2014, MNRAS, 444, 43

Grier, C. J., Peterson, B. M., Horne, K., et al. 2013, ApJ, 764, 47

Hall, P. B., Hutsemékers, D., Anderson, S. F., et al. 2003, ApJ, 593, 189

Hewett, P. C., \& Wild, V. 2010, MNRAS, 405, 2302

Hu, C., Wang, J.-M., Ho, L. C., et al. 2008, ApJ, 683, L115

Hu, C., Wang, J.-M., Ho, L. C., et al. 2012, ApJ, 760, 126

Karouzos, M., Woo, J.-H., Matsuoka, K., et al. 2015, ApJ, 815, 128

Kaspi, S., Brandt, W. N., Maoz, D., et al. 2007, ApJ, 659, 997

Kelly, B. C., \& Bechtold, J. 2007, ApJS, 168, 1

Kollatschny, W., \& Zetzl, M. 2013, A\&A, 549, A100

Kriss, G. 1994, ASP Conf. Ser., 61, 437

Kuraszkiewicz, J., Wilkes, B. J., Schmidt, G., et al. 2009, ApJ, 692, 1180

Kwan, J., \& Krolik, J. H. 1981, ApJ, 250, 478

Laor, A., Fiore, F., Elvis, M., Wilkes, B. J., \& McDowell, J. C. 1997, ApJ, 477, 93

Latif, M. A., \& Ferrara, A. 2016, PASA, 33, e051

Leighly, K. M. 2004, ApJ, 611, 125

Lira, P., Botti, I., Kaspi, S., \& Netzer, H. 2017, Front. Astron. Space Sci., 4, 71

Lira, P., Kaspi, S., Netzer, H., et al. 2018, ApJ, 865, 56

Martínez-Aldama, M. L., Dultzin, D., Marziani, P., et al. 2015, ApJS, 217, 3

Martínez-Aldama, M. L., Del Olmo, A., Marziani, P., et al. 2018, Front. Astron. Space Sci., 4, 65

Marziani, P., Sulentic, J. W., Dultzin-Hacyan, D., Calvani, M., \& Moles, M. 1996, ApJS, 104, 37

Marziani, P., Sulentic, J. W., Zamanov, R., et al. 2003, ApJS, 145, 199 
Marziani, P., Sulentic, J. W., Negrete, C. A., et al. 2010, MNRAS, 409, 1033

Marziani, P., Sulentic, J. W., Plauchu-Frayn, I., \& del Olmo, A. 2013a, ApJ, 764 150

Marziani, P., Sulentic, J. W., Plauchu-Frayn, I., \& del Olmo, A. 2013b, A\&A, 555, A89

Marziani, P., Martínez Carballo, M. A., Sulentic, J. W., et al. 2016, Ap\&SS, 361, 29

Marziani, P., Dultzin, D., Sulentic, J. W., et al. 2018, Front. Astron. Space Sci. 5,6

Matsuoka, Y., Kawara, K., \& Oyabu, S. 2008, ApJ, 673, 62

Matsuoka, K., Silverman, J. D., Schramm, M., et al. 2013, ApJ, 771, 64

McLure, R. J., \& Dunlop, J. S. 2004, MNRAS, 352, 1390

McLure, R. J., \& Jarvis, M. J. 2002, MNRAS, 337, 109

Mejía-Restrepo, J. E., Trakhtenbrot, B., Lira, P., Netzer, H., \& Capellupo, D. M. 2016, MNRAS, 460, 187

Mejía-Restrepo, J. E., Lira, P., Netzer, H., Trakhtenbrot, B., \& Capellupo, D. 2017, Front. Astron. Space Sci., 4, 70

Mejía-Restrepo, J. E., Lira, P., Netzer, H., Trakhtenbrot, B., \& Capellupo, D. M. 2018a, Nat. Astron., 2, 63

Mejía-Restrepo, J. E., Trakhtenbrot, B., Lira, P., \& Netzer, H. 2018b, MNRAS, 478, 1929

Negrete, C. A., Dultzin, D., Marziani, P., \& Sulentic, J. W. 2014, ApJ, 794, 95

Negrete, C. A., Dultzin, D., Marziani, P., et al. 2017, Front. Astron. Space Sci. 4,59

Negrete, C. A., Dultzin, D., Marziani, P., et al. 2018, A\&A, 620, A118

Netzer, H. 1990, in Active Galactic Nuclei, eds. R. D. Blandford, H. Netzer, L. Woltjer, T. J.-L. Courvoisier, \& M. Mayor, 57

Netzer, H., \& Marziani, P. 2010, ApJ, 724, 318

Netzer, H., Lira, P., Trakhtenbrot, B., Shemmer, O., \& Cury, I. 2007, ApJ, 671, 1256

Osterbrock, D. E., \& Shuder, J. M. 1982, ApJS, 49, 149

Park, D., Woo, J.-H., Denney, K. D., \& Shin, J. 2013, ApJ, 770, 87

Pei, L., Fausnaugh, M. M., Barth, A. J., et al. 2017, ApJ, 837, 131

Peterson, B. M. 2014, SpScieRev, 183, 253

Peterson, B. M., \& Ferland, G. J. 1986, Nature, 324, 345

Peterson, B. M., \& Wandel, A. 1999, ApJ, 521, L95

Peterson, B. M., \& Wandel, A. 2000, ApJ, 540, L13

Peterson, B. M., Bentz, M. C., Desroches, L.-B., et al. 2005, ApJ, 632, 799

Plotkin, R. M., Shemmer, O., Trakhtenbrot, B., et al. 2015, ApJ, 805, 123

Proga, D., \& Kallman, T. R. 2004, ApJ, 616, 688

Proga, D., Stone, J. M., \& Kallman, T. R. 2000, ApJ, 543, 686

Punsly, B. 2010, ApJ, 713, 232

Punsly, B., \& Zhang, S. 2011, ApJ, 735, L3

Richards, G. T., Kruczek, N. E., Gallagher, S. C., et al. 2011, AJ, 141, 167

Runnoe, J. C., Brotherton, M. S., DiPompeo, M. A., \& Shang, Z. 2014, MNRAS, 438,3263

Saito, Y., Imanishi, M., Minowa, Y., et al. 2016, PASJ, 68, 1

Sdowski, A., Narayan, R., McKinney, J. C., \& Tchekhovskoy, A. 2014, MNRAS, 439, 503

Shang, Z., Brotherton, M. S., Wills, B. J., et al. 2011, ApJS, 196, 2

Shen, Y. 2013, Bull. Astron. Soc. India, 41, 61

Shen, Y. 2016, ApJ, 817, 55

Shen, Y., \& Ho, L. C. 2014, Nature, 513, 210

Shen, Y., \& Liu, X. 2012, ApJ, 753, 125

Shen, Y., Brandt, W. N., Richards, G. T., et al. 2016, ApJ, 831, 7
Snedden, S., \& Gaskell, C. 2004, in AGN Physics with the Sloan Digital Sky

Survey, eds. G. T. Richards, \& P. B. Hall, ASP Conf. Ser., 311, 197

Snedden, S. A., \& Gaskell, C. M. 2007, ApJ, 669, 126

Steinhardt, C. L., \& Silverman, J. D. 2013, PASJ, 65, 82

Stevens, I. R., \& Kallman, T. R. 1990, ApJ, 365, 321

Storchi-Bergmann, T., Schimoia, J. S., Peterson, B. M., et al. 2017, ApJ, 835, 236

Strateva, I. V., Strauss, M. A., Hao, L., et al. 2003, AJ, 126, 1720

Sulentic, J. W. 1989, ApJ, 343, 54

Sulentic, J., \& Marziani, P. 2015, Front. Astron. Space Sci., 2, 6

Sulentic, J. W., Marziani, P., \& Dultzin-Hacyan, D. 2000a, ARA\&A, 38, 521

Sulentic, J. W., Marziani, P., Zwitter, T., Dultzin-Hacyan, D., \& Calvani, M. 2000b, ApJ, 545, L15

Sulentic, J. W., Zwitter, T., Marziani, P., \& Dultzin-Hacyan, D. 2000c, ApJ, 536, L5

Sulentic, J. W., Marziani, P., Zamanov, R., et al. 2002, ApJ, 566, L71

Sulentic, J. W., Stirpe, G. M., Marziani, P., et al. 2004, A\&A, 423, 121

Sulentic, J. W., Repetto, P., Stirpe, G. M., et al. 2006a, A\&A, 456, 929

Sulentic, J. W., Dultzin-Hacyan, D., Marziani, P., et al. 2006b, Rev. Mex. Astron. Astrofis., 42, 23

Sulentic, J. W., Bachev, R., Marziani, P., Negrete, C. A., \& Dultzin, D. 2007, ApJ, 666, 757

Sulentic, J., Marziani, P., \& Zamfir, S. 2011, Balt. Astron., 20, 427

Sulentic, J. W., Marziani, P., del Olmo, A., et al. 2014, A\&A, 570, A96

Sulentic, J. W., Martínez-Carballo, M. A., Marziani, P., et al. 2015, MNRAS, 450, 1916

Sulentic, J. W., del Olmo, A., Marziani, P., et al. 2017, A\&A, 608, A122

Sun, J., \& Shen, Y. 2015, ApJ, 804, L15

Sun, M., Xue, Y., Richards, G. T., et al. 2018, ApJ, 854, 128

Tilton, E. M., \& Shull, J. M. 2013, ApJ, 774, 67

Trakhtenbrot, B., \& Netzer, H. 2012, MNRAS, 427, 3081

Trevese, D., Perna, M., Vagnetti, F., Saturni, F. G., \& Dadina, M. 2014, ApJ, 795, 164

Tytler, D., \& Fan, X.-M. 1992, ApJS, 79, 1

Vanden Berk, D. E., Richards, G. T., Bauer, A., et al. 2001, AJ, 122, 549

Vestergaard, M. 2003, ApJ, 599, 116

Vestergaard, M., \& Peterson, B. M. 2006, ApJ, 641, 689

Vietri, G. 2017, Am. Astron. Soc. Meeting Abstracts, 229, 302.06

Vietri, G., Piconcelli, E., Bischetti, M., et al. 2018, A\&A, 617, A81

Vollmer, B., Schartmann, M., Burtscher, L., et al. 2018, A\&A, 615, A164

Wang, J., \& Li, Y. 2011, ApJ, 742, L12

Wang, T., Brinkmann, W., \& Bergeron, J. 1996, A\&Ap, 309, 81

Wang, J., Dong, X., Wang, T., et al. 2009, ApJ, 707, 1334

Wang, H., Wang, T., Zhou, H., et al. 2011, ApJ, 738, 85

Wang, J.-M., Qiu, J., Du, P., \& Ho, L. C. 2014a, ApJ, 797, 65

Wang, J.-M., Du, P., Li, Y.-R., et al. 2014b, ApJ, 792, L13

Wang, J.-M., Du, P., Brotherton, M. S., et al. 2017, Nat. Astron., 1, 775

Wills, B. J., Netzer, H., \& Wills, D. 1985, ApJ, 288, 94

Wills, B. J., Brotherton, M. S., Fang, D., Steidel, C. C., \& Sargent, W. L. W. 1993, ApJ, 415, 563

Wisotzki, L., Christlieb, N., Bade, N., et al. 2000, A\&A, 358, 77

Yip, C. W., Connolly, A. J., Vanden Berk, D. E., et al. 2004, AJ, 128, 2603

Yong, S. Y., King, A. L., Webster, R. L., et al. 2018, MNRAS, 479, 4153

Zamanov, R., Marziani, P., Sulentic, J. W., et al. 2002, ApJ, 576, L9

Zamfir, S., Sulentic, J. W., Marziani, P., \& Dultzin, D. 2010, MNRAS, 403, 1759 
Appendix A: The MS / 4DE1 formalism: a glossary

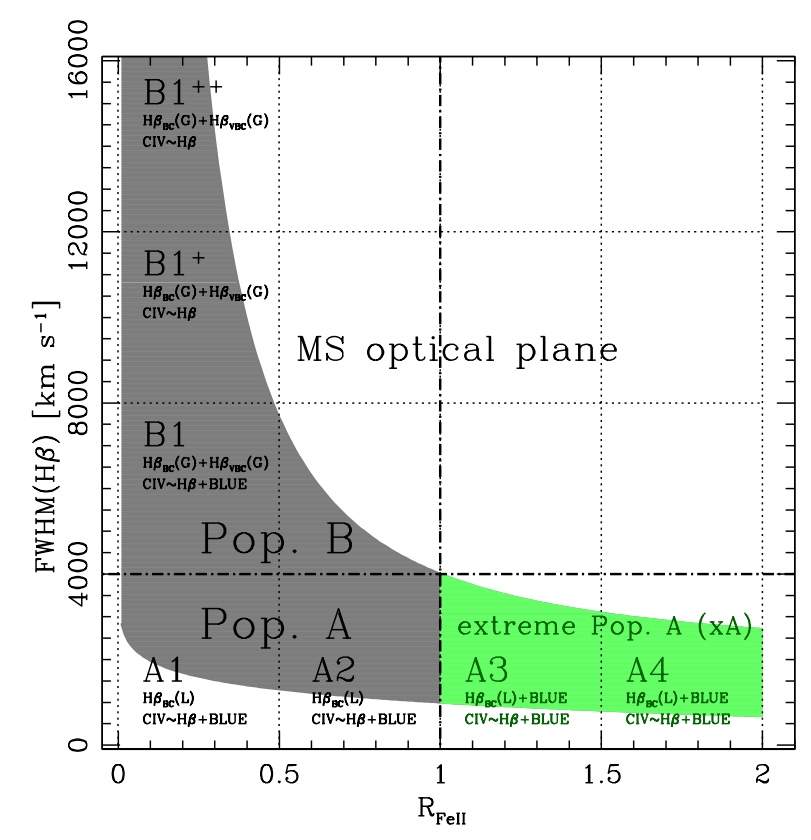

Fig. A.1. Schematic representation of the optical plane of the quasar MS, with the subdivisions identifying spectral types along the sequence. The main components that are blended in the $\mathrm{H} \beta$ and Civ $\lambda 1549$ profile are listed in each spectral bin. The shaded area shows the approximate occupation of low- $z$ quasars sample in the plane.

In the optical plane of the quasar main sequence, spectral types are isolated following Sulentic et al. (2002). Figure A.1 provides a sketch and Table A.1 a summary with the spectral types identification in the optical plane of the MS. Here we provide a glossary of the MS-related terms and acronyms employed in the paper, along the order of the quasar main sequence. A more thorough description can be found in Sulentic et al. (2011) and Marziani et al. (2018).

The rational for two type-1 quasar populations (A and B) was originally given by Sulentic et al. (2000a). Spectral types A3 and $\mathrm{A} 4$ are also indicated as extreme Population $\mathrm{A}$, with $R_{\mathrm{FeII}} \gtrsim 1$. They are the highest radiators per unit mass and possibly superEddington accretors (Wang et al. 2014b).

The 4D Eigenvector-1 formalism was introduced to limit the set of MS-correlated parameters to the four ones that are most relevant for the MS physical interpretation. In addition to FWHM $\mathrm{H} \beta, R_{\mathrm{FeII}}$, and $c\left(\frac{1}{2}\right) \mathrm{CIV} \lambda 1549$, the soft X-ray photon index $\Gamma_{\text {soft }}$ is also considered (Sulentic et al. 2000b). The four parameters were meant to represent the velocity dispersion of the LIL-emitting part of the BLR, the physical condition within the LIL-BLR $\left(R_{\mathrm{FeII}}\right)$, the dynamical condition of the HIL-emitting gas, and the accretion state of the black hole. $\Gamma_{\text {soft }}>2$ implies a soft X-ray excess that is exclusive of Population A (Wang et al. 1996; Boller et al. 1996; Shen \& Ho 2014; Bensch et al. 2015).

Line components assumed in the decomposition along the MS are defined as follows (see also the sketch of Fig. A.1 and Table A.2).

BLUE becomes detectable as a blueward excess in the $\mathrm{H} \beta$ profile mainly in A3 and A4 (more infrequently in A2). For CIv $\lambda 1549$, BLUE increases in prominence along the sequence from $\mathrm{B} 1^{++}$, to $\mathrm{A} 4$. In $\mathrm{B} 1^{++}$and $\mathrm{B} 1^{+}$is weak and often undetectable, while in A3 and A4 it may dominate Civ $\lambda 1549$ emission.
Table A.1. Spectral type definition along the main sequence.

\begin{tabular}{|c|c|}
\hline ST & Definition \\
\hline \multicolumn{2}{|c|}{$\begin{array}{l}\text { Population B: } F W H M(\mathrm{H} \beta) \gtrsim 4000 \mathrm{~km} \mathrm{~s}^{-1} \text {. Virial- } \\
\text { dominated with redward-asymmetric profiles in } \mathrm{H} \beta \text { and } \\
\text { MgII } \lambda 2800 \text {. Also described a disk-dominated (Richards } \\
\text { et al. 2011). }\end{array}$} \\
\hline $\mathrm{B} 1++$ & $\begin{array}{l}12000 \mathrm{~km} \mathrm{~s}^{-1} \leq F W H M(\mathrm{H} \beta)<16000 \mathrm{~km} \mathrm{~s}^{-1}, \\
R_{\mathrm{FeII}}<0.5\end{array}$ \\
\hline $\mathrm{B} 1+$ & $\begin{array}{l}8000 \mathrm{~km} \mathrm{~s}^{-1} \leq F W H M(\mathrm{H} \beta)<12000 \mathrm{~km} \mathrm{~s}^{-1}, \\
R_{\mathrm{FeII}}<0.5\end{array}$ \\
\hline B1 & $\begin{array}{l}4000 \mathrm{~km} \mathrm{~s}^{-1} \leq F W H M(\mathrm{H} \beta)<8000 \mathrm{~km} \mathrm{~s}^{-1}, \\
R_{\mathrm{FeII}}<0.5\end{array}$ \\
\hline \multicolumn{2}{|c|}{$\begin{array}{l}\text { Population A: } F W H M(\mathrm{H} \beta) \lesssim 4000 \mathrm{~km} \mathrm{~s}^{-1} \text {. Sources fre- } \\
\text { quently show CIV } \lambda 1549 \text { blueshifts, and } \mathrm{H} \beta \text { Lorentzian- } \\
\text { like profiles (Du et al. 2016). Includes a range of FeII } \\
\text { emission. }\end{array}$} \\
\hline A1 & $F W H M(\mathrm{H} \beta)<4000 \mathrm{~km} \mathrm{~s}^{-1} ; R_{\mathrm{FeII}}<0.5$ \\
\hline $\mathrm{A} 2$ & $F W H M(\mathrm{H} \beta)<4000 \mathrm{~km} \mathrm{~s}^{-1} ; \leq 0.5 R_{\mathrm{FeII}}<1$ \\
\hline $\begin{array}{l}\text { A3 } \\
{[\mathrm{xA}]}\end{array}$ & $F W H M(\mathrm{H} \beta)<4000 \mathrm{~km} \mathrm{~s}^{-1} ; \leq 1 R_{\mathrm{FeII}}<1.5$ \\
\hline $\begin{array}{l}\mathrm{A} 4 \\
{[\mathrm{xA}]}\end{array}$ & $F W H M(\mathrm{H} \beta)<4000 \mathrm{~km} \mathrm{~s}^{-1} ; 1.5 \leq R_{\mathrm{FeII}}<2$ \\
\hline
\end{tabular}

Table A.2. Line components along the main sequence.

\begin{tabular}{|c|c|}
\hline $\begin{array}{l}\text { Line com- } \\
\text { ponent }\end{array}$ & Definition \\
\hline \multicolumn{2}{|r|}{ Low-ionization lines (LILs): $\mathrm{HI} \mathrm{H} \beta$} \\
\hline $\mathrm{H} \beta_{\mathrm{VBC}}$ & $\begin{array}{l}\text { Gaussian } F W H M \sim 10000 \mathrm{~km} \mathrm{~s}^{-1} \text {, redshifted } \\
\text { by } 1-2000 \mathrm{~km} \mathrm{~s}^{-1} \text { (defining property of Pop. } \\
\text { B; absent in Pop. A) }\end{array}$ \\
\hline $\mathrm{H} \beta_{\mathrm{BC}}$ & $\begin{array}{l}\text { Lorentzian, } F W H M \sim 1-4000 \mathrm{~km} \mathrm{~s}^{-1} \text { (Pop. } \\
\text { A); Gaussian } F W H M \mathrm{H} \beta \gtrsim 4000 \mathrm{~km} \mathrm{~s}^{-1} \text { (Pop. } \\
\text { B) }\end{array}$ \\
\hline $\mathrm{H} \beta$ BLUE & $\begin{array}{l}\text { Asymmetric Gaussian which models an excess } \\
\text { of emission on the blue side of } \mathrm{H} \beta \text {; usually } \\
\text { weak save in ST A } 3 \text { and A } 4\end{array}$ \\
\hline $\mathrm{H} \beta$ & $\begin{array}{l}\text { Sum of } \mathrm{H} \beta_{\mathrm{BC}}, \mathrm{H} \beta_{\mathrm{VBC}} \text {, and BLUE (when appli- } \\
\text { cable); full broad } \mathrm{H} \beta \text { profile }\end{array}$ \\
\hline $\mathrm{H} \beta_{\mathrm{NC}}$ & $\mathrm{H} \beta$ narrow component \\
\hline
\end{tabular}
High-ionization lines (HILs): Civ $\lambda 1549$

Civ $11549_{\mathrm{VBC}}$ Gaussian $F W H M \sim 10000 \mathrm{~km} \mathrm{~s}^{-1}$, redshifted by $1-2000 \mathrm{~km} \mathrm{~s}^{-1}$

Civ $\lambda 1549_{\mathrm{BC}} \quad$ Lorentzian, if $F W H M(\mathrm{H} \beta) \lesssim 4000 \mathrm{~km} \mathrm{~s}^{-1}$ (Pop. A); Gaussian FWHM $\gtrsim 4000 \mathrm{~km} \mathrm{~s}^{-1}$ (Pop. B)

Civ 21549 Asymmetric Gaussian which models an excess BLUE of emission on the blue side of Civ $\lambda 1549$; detected in most quasar and most prominent in spectral types A3 and A4

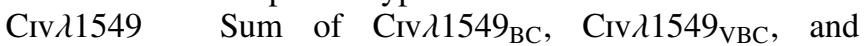
Civ $\lambda 1549$ BLUE; full broad Civ $\lambda 1549$ profile

Civ $\lambda 1549_{\mathrm{NC}}$ Civ $\lambda 1549$ narrow component, prominent in Pop. B at low-z; almost absent in most Pop. A sources 


\section{Appendix B: Effect of orientation on $\mathrm{H} \beta M_{\mathrm{BH}}$ estimates}

From the inversion of Eq. (9), we obtain an expression for the viewing angle $\theta=\arcsin \sqrt{x^{2} / 4-\kappa^{2}}$, where $x=\delta v_{\mathrm{obs}} / \delta v_{\mathrm{K}}$ and $\kappa=\delta v_{\text {iso }} / \delta v_{\mathrm{K}}$. The probability to observe $\delta v_{\mathrm{obs}}$ for a given $\delta v_{\mathrm{K}}$ is then

$$
P(x)=\sqrt{x^{2} / 4-\kappa^{2}} \frac{\mathrm{d} \theta}{\mathrm{d} x}=\frac{x / 4}{\sqrt{\kappa^{2}-x^{2} / 4+1}} .
$$

The black hole mass is $\propto x^{2}$. Therefore the average effect can be written as

$$
\left\langle\frac{M_{\mathrm{BH}, \mathrm{obs}}}{M_{\mathrm{BH}, \mathrm{K}}}\right\rangle=\int_{x\left(v_{\text {iso }}\right)}^{x \text { (edge })} x^{2} P(x) \mathrm{d} x / \int_{x\left(v_{\text {iso }}\right)}^{x(\text { edge })} P(x) \mathrm{d} x .
$$

The integrals of Eq. (B.2) can be computed analytically:

$$
\begin{aligned}
\int x^{2} P(x) \mathrm{d} x= & \left(-\frac{4}{3} \sqrt{4 \kappa^{2}-x^{2}+4}\right) \\
& -\frac{4}{3} \kappa^{2} \sqrt{4 \kappa^{2}-x^{2}+4}-\frac{1}{6}\left(x^{2} \sqrt{4 \kappa^{2}-x^{2}+4}\right),
\end{aligned}
$$

$\int P(x) \mathrm{d} x=-\sqrt{1+\kappa^{2}-x^{2} / 4}$

We note that the integration limits in $x$ (which correspond to $\theta=0$ and $\theta=45$ ) are different for $\kappa=0.1$ and 0.5 . For $\kappa=0.1$, $x_{\text {iso }}=0.2$ and $x_{\text {edge }} \approx 1.43$ In the latter case, $\theta=0$ corresponds to $x_{\min }=1$ and $\theta=45$ to $x_{\text {edge }} \approx 1.73: M_{\mathrm{BH}}$ will be always overestimated, for every possible $\theta$ value larger than 0 .

If we consider the Eddington ratio, we obtain

$$
\int P(x) / x^{2} \mathrm{~d} x=-\arctan \mathrm{h}\left(\frac{\sqrt{\kappa^{2}+1}}{\sqrt{\kappa^{2}-x^{2} / 4+1}}\right) / 4 \sqrt{\kappa^{2}+1} .
$$

Equation (B.5) implies a significant effect on $L / L_{\mathrm{Edd}}$ for both $\kappa=0.1$ and $\kappa=0.5$. In the first case, the $L / L_{\text {Edd }}$ will be overestimated by a factor $\approx 1.8$. In the second case, the $L / L_{\text {Edd }}$ will be underestimated by a factor $\approx 2$, due to the systematic overestimation in $M_{\mathrm{BH}}$.

\section{Appendix C: Origin of scatter in scaling laws}

We considered synthetic samples of $\sim 10000$ objects obtained from random variates with distribution $P(\theta) \propto \sin \theta(0 \leq$ $\theta \leq \pi / 4)$, and "true" $M_{\mathrm{BH}}$ uniformly distributed in the range $10^{7} M_{\odot} \leq M_{\mathrm{BH}} \leq 10^{9} M_{\odot}$. The dependence on orientation of the $\mathrm{H} \beta \mathrm{FWHM}$ is assumed to follow Eq. (9), with $\kappa=0.1$. The effect of orientation on FWHM is such that in a randomly oriented synthetic sample the $M_{\mathrm{BH}}$ estimated from $\mathrm{H} \beta$ deviates from the true $M_{\mathrm{BH}}$ as in Fig. C. 1 (top panel). The dispersion is $\approx 0.35$, which is comparable to the uncertainty in the scaling-law $M_{\mathrm{BH}}$ estimate following Vestergaard \& Peterson $(2006, \approx 0.5$ at $1 \sigma$ confidence level).

The estimates of $M_{\mathrm{BH}}$ CIV $\lambda 1549$ show a significant scatter if plotted against $M_{\mathrm{BH}} \mathrm{H} \beta$ (Fig. 12). The origin of the scatter is in part related to orientation, in part to the outflow component. The second panel from top of Fig. C. 1 shows the $M_{\mathrm{BH}}$ Civ $\lambda 1549$ vs. $\mathrm{H} \beta$ for a synthetic sample to which no correction has been applied. FWHM of Civ $\lambda 1549$ and $\mathrm{H} \beta$ are expected to be related to the Keplerian velocity by Eqs. (12) and (10), respectively. The
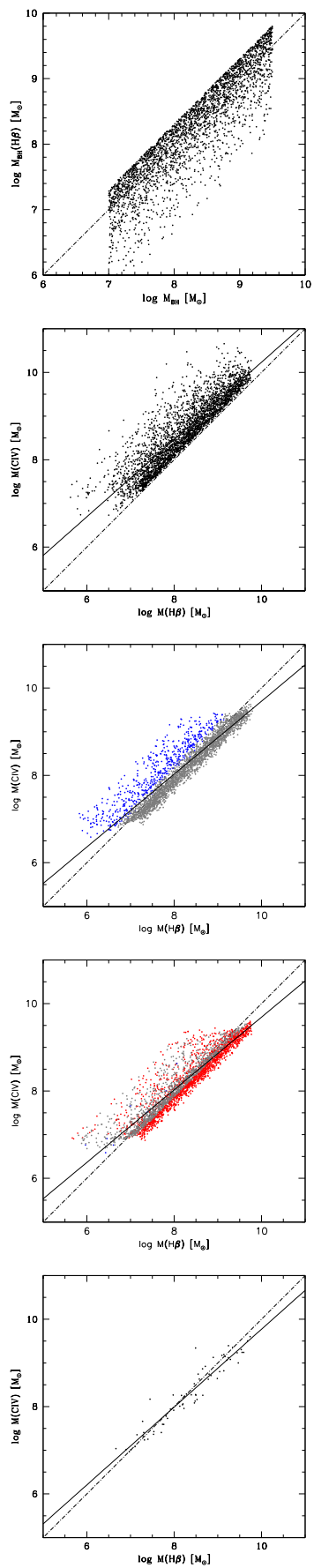

Fig. C.1. Top: $M_{\mathrm{BH}}$ with effect of orientation via Eq. (10) vs. the "true" $M_{\mathrm{BH}}$, for a synthetic sample of 10000 sources. Second from top: $M_{\mathrm{BH}}$ Civ $\lambda 1549$ vs. $M_{\mathrm{BH}} \mathrm{H} \beta$ estimated for an Eddington ratio distribution as described in the text and no correction. Third from top: FWHM $M_{\mathrm{BH}}$ CIv $\lambda 1549$ corrected because of outflow broadening using a correction factor $\xi$. The blue dots identify the $M_{\mathrm{BH}} \mathrm{H} \beta$ estimates that are under 0.33 dex the true $M_{\mathrm{BH}}$; the red ones are for overestimates by more than 0.33 dex. The gray dots represent mass estimates within -0.33 and +0.33 dex from the true value. Fourth from top: as above but with color-coding referring to Civ $\lambda 1549 M_{\mathrm{BH}}$. Bottom: synthetic sample with $n \lesssim 80$ sources, as in the FOS+HE sample. See text for more details. In all panels, the dot-dashed line is the equality line; the filled line traces an unweighted least-squares fit.

distribution of $L / L_{\text {Edd }}$ has been assumed Gaussian, peaking at $\log L / L_{\mathrm{Edd}}=-0.3$, and $\sigma \approx 0.5$. Typical values of $\xi_{\mathrm{CIV}}$ and $Q$ are appropriate for Pop. A sources. There is a strong bias $(0.5 \mathrm{dex})$ 
and a standard deviation of the mass ratios of $\approx 0.6 \mathrm{dex}$. In some rare instances (a combination of face-on orientation and large outflow velocity) the ratio between $M_{\mathrm{BH}}$ from $\mathrm{H} \beta$ and Civ $\lambda 1549$ can reach a factor $\sim 10^{2}$, as actually found by Sulentic et al. (2007).

The third and fourth panels from the top show the same configuration, but after applying a correction factor $\xi_{\mathrm{CIV}}$ in the form $1 / \zeta\left(L, L / L_{\mathrm{Edd}}\right)=1 /\left(1+k L^{a}\left(L / L_{\mathrm{Edd}}\right)^{b}\right)$, with $a \approx 0.1, b \approx 1$ and no dependence on orientation, intended to mimic the correction actually applied to the data in Sect. 4. The orientation effect is changing the CIV $\lambda 1549$ FWHM following Eq. (12), and therefore displacing the $M_{\mathrm{BH}}$ from the true mass also after correcting the FWHM. We note that the outlying blue points are due to $\mathrm{H} \beta$ underestimates of the mass by more than 0.33 dex because of low values of the viewing angle $\theta$. On the contrary, the relatively high value of $Q$ in the simulation produces a significant fraction of $M_{\mathrm{BH}}$ CIV $\lambda 1549$ which are overestimating the $M_{\mathrm{BH}}$ by more than $0.33 \mathrm{dex}$ (red points). The dispersion is however reduced with respect to the case with no correction, with an $\mathrm{rms} \approx 0.3$. The bottom panel is a realization of the synthetic sample for a number of sources $\lesssim 100$, comparable to the size of the FOS $+\mathrm{HE}$ sample, and Gaussian distribution of $L / L_{\text {Edd }}$ as for case shown and correction in the second panel from top.

In all of these cases save the one of the second panel from top the dispersion remains $\sim 0.3$, comparable to the one measured for the scaling laws of Eqs. (17) and (18). It is interesting to note that in the framework of the toy model, if $Q=0$ the orientation-induced scatter is the same for $\mathrm{H} \beta$ and Civ $\lambda 1549$; if $Q \approx 4$, Civ $\lambda 1549$ becomes an almost perfect VBE, with all the scatter being due to $\mathrm{H} \beta$, in a plot $M_{\mathrm{BH}} \mathrm{CIV} \lambda 1549$ vs $M_{\mathrm{BH}} \mathrm{H} \beta$. These results may be consistent with no strong dependence on orientation of the Civ $\lambda 1549$ line shift in RL quasars (Runnoe et al. 2014) $)^{3}$.

A uniform distribution of $Q$ between 0 and 1.6, a situation more appropriate for Pop. B, was also considered. The results were similar with smaller dispersion and biases.
3 However, it is not clear whether the results of Runnoe et al. (2014) are applicable to radio quiet quasars: RL sources show no strong evidence of large blueshifts (Sulentic et al. 2007; Richards et al. 2011) as the disk outflow properties may be strongly affected by the powerful radio ejecta (e.g. Punsly 2010; Punsly \& Zhang 2011; Sulentic et al. 2015, and references therein). 